\title{
WELL-POINTED COALGEBRAS
}

\author{
JIŘí ADÁMEK ${ }^{a}$, STEFAN MILIUS $^{b}$, LAWRENCE S. MOSS $^{c}$, AND LURDES SOUSA $^{d}$ \\ ${ }^{a}$ Institut für Theoretische Informatik, Technische Universität Braunschweig, Germany \\ e-mail address: adamek@iti.cs.tu-bs.de \\ ${ }^{b}$ Lehrstuhl für Theoretische Informatik, Friedrich-Alexander Universität Erlangen-Nürnberg, Ger- \\ many \\ e-mail address: mail@stefan-milius.eu \\ ${ }^{c}$ Department of Mathematics, Indiana University, Bloomington, IN, USA \\ e-mail address: lsm@cs.indiana.edu \\ ${ }^{d}$ Polytechnic Institute of Viseu, Portugal \& Centre for Mathematics of the University of Coimbra, \\ Portugal \\ e-mail address: sousa@mat.estv.ipv.pt
}

\begin{abstract}
For endofunctors of varieties preserving intersections, a new description of the final coalgebra and the initial algebra is presented: the former consists of all well-pointed coalgebras. These are the pointed coalgebras having no proper subobject and no proper quotient. The initial algebra consists of all well-pointed coalgebras that are well-founded in the sense of Osius [22] and Taylor 29]. And initial algebras are precisely the final well-founded coalgebras. Finally, the initial iterative algebra consists of all finite wellpointed coalgebras. Numerous examples are discussed e.g. automata, graphs, and labeled transition systems.
\end{abstract}

\section{INTRODUCTION}

Initial algebras are known to be of primary interest in denotational semantics, where abstract data types are often presented as initial algebras for an endofunctor $H$ expressing the type of the constructor operations of the data type. For example, finite binary trees are the initial algebra for the functor $H X=X \times X+1$ on sets. Analogously, final coalgebras for an endofunctor $H$ play an important role in the theory of systems developed by Rutten [23]: $H$ expresses the system type, i. e., which kind of one-step reactions states can exhibit (input, output, state transitions etc.), and the coalgebras for $H$ are precisely systems with a set of states having reactions of type $H$. The elements of a final coalgebra represent the behavior

2012 ACM CCS: [Theory of computation]:Models of computation; Formal languages and automata theory.

Key words and phrases: Well-founded coalgebra, well-pointed coalgebra, initial algebra, final coalgebra, iterative algebra.

${ }^{d}$ Financial support by CMUC/FCT (Portugal) and the FCT Grant PTDC/MAT/120222/2010 is acknowledged by the last author. 
of all states in all systems of type $H$, and the unique homomorphism from a system into the final one assigns to every state its behavior. For example, deterministic automata with input alphabet $I$ are coalgebras for $H X=X^{I} \times\{0,1\}$, and the final coalgebra is the set of all languages on $I$.

In this paper a unified description is presented for (a) initial algebras, (b) final coalgebras and (c) initial iterative algebras (in the automata example this is the set of all regular languages on $I$ ). We also demonstrate that this new description provides a unifying view of a number of important examples. We first work with set functors $H$ preserving intersections. This is an extremely mild requirement that most "everyday" set functors satisfy, see Example 3.2. We prove that the final coalgebra for $H$ can then be described as the set of all well-pointed coalgebras, i.e., pointed coalgebras not having any proper subobject and also not having any proper quotient. Moreover, the initial algebra can be described as the set of all well-pointed coalgebras which are well-founded in the sense of Osius 22 ] and Taylor $[28,29$. We then extend these results to all endofunctors of varieties preserving intersections.

Before we mention the definition of well-founded coalgebra, recall that the notion of well-foundedness of relations $R \subseteq X \times X$ has several alternative forms:

(1) No proper subset $Y$ of $X$ has the property that if all $R$-successors of a given point $x \in X$ lie in $Y$, then $x \in Y$ as well.

(2) There is no infinite path $x_{0} R x_{1} R x_{2} R \cdots$.

(3) There is a map rk from $X$ to ordinals such that $\mathrm{rk}(x)>\operatorname{rk}(y)$ whenever $x R y$.

For sets and relations as usual, these conditions are equivalent. The first of these is an induction principle, and this is closest to what we are calling well-foundedness in this paper, following Taylor. The equivalence of the first and the second requires Dependent Choice, a weak form of the Axiom of Choice; in any case, our work in this area does not use this at all. The last condition is close to a result which we will see, but note as well that even this requires something special about sets, namely the Replacement Axiom.

The notion of well-foundedness of a coalgebra $(A, \alpha)$ generalizes condition (1) above. It says that no proper subcoalgebra $m:\left(A^{\prime}, \alpha^{\prime}\right) \hookrightarrow(A, \alpha)$ forms a pullback

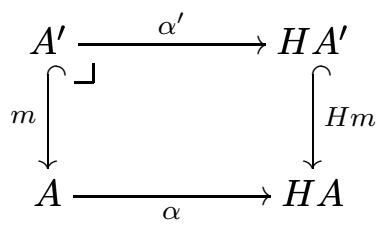

This concept was first studied by Osius [22] for graphs considered as coalgebras for the power-set functor $\mathscr{P}$ : a graph is well-founded in the coalgebraic sense iff it is well-founded in any of the equivalent senses above. Taylor [28, 29] introduced well-founded coalgebras for general endofunctors, and he proved that for set functors preserving inverse images the concepts of initial algebra and final well-founded coalgebra coincide.

Returning to our topic, we are going to prove that for every set functor $H$ the concepts of initial algebra and final well-founded coalgebra coincide; the step towards making no assumptions on $H$ is non-trivial. We also prove the same result for endofunctors of locally finitely presentable categories preserving finite intersections. And if $H$ preserves (wide) intersections, we describe its final coalgebra and initial algebra using well-pointed coalgebras. 
The last section takes a number of known important special cases: deterministic (Mealy and Moore) automata, trees, labeled transition systems, non-well-founded sets, etc., and demonstrates how well-pointed coalgebras work in each case. Here we describe, in every example, besides the initial algebra and the final coalgebra, the initial iterative algebra [6] (equivalently, final locally finite coalgebra, see [20, 10]) as the set of all finite well-pointed coalgebras.

\section{WELL-FOUNDED COALGEBRAS}

In this section we recall the concept of well-founded coalgebra of Osius [22] and Taylor [28]. Our main result is that

$$
\text { initial algebra }=\text { final well-founded coalgebra }
$$

holds for all endofunctors of Set. (In the case where the endofunctor preserves inverse images, this result can be found in [28].) For more general categories the result above holds whenever the endofunctor preserves finite intersections.

\subsection{Well-founded coalgebras in locally finitely presentable categories.}

We make several assumptions on the base category $\mathscr{A}$ in our study.

\section{Definition 2.1.}

(1) A category $\mathscr{A}$ is locally finitely presentable (LFP) if

(a) $\mathscr{A}$ is complete;

(b) there is a set of finitely presentable objects whose closure under filtered colimits is all of $\mathscr{A}$.

(See [15] or [8] for more on LFP categories.)

(2) An object $A$ of (any category) $\mathscr{A}$ is called simple if every strong epimorphism (see Remark 2.6) with domain $A$ is invertible. (In categories with (strong epi, mono)factorizations, see Remark 2.6, this is equivalent to saying that every morphism with domain $A$ is a monomorphism.)

Remark 2.2. The concept of simple object stems from general Algebra, where strong epimorphisms are precisely the surjective homomorphisms, thus, an algebra is simple iff it has no nontrivial congruence.

Assumption 2.3. Throughout this section our base category $\mathscr{A}$ is locally finitely presentable and has a simple initial object 0 .

Examples 2.4. The categories of sets, graphs, posets, and semigroups are locally finitely presentable. The initial objects of these categories are empty, hence simple. The LFP category of rings has the initial object $\mathbb{Z}$ that is not simple.

Notation 2.5. For every endofunctor $H$ denote by

\section{Coalg $H$}

the category of coalgebras $\alpha: A \longrightarrow H A$ and coalgebra homomorphisms.

Remark 2.6. There are some consequences of the LFP assumption that play an important role in our development: 
1. $\mathscr{A}$ has (strong epi, mono)-factorizations, see [8, Proposition 1.16]. (Recall that an epimorphism $e$ is called strong if it fulfils the diagonal fill-in property w. r. t. all monomorphisms, i.e., $f e=m g$ with $m$ a monomorphism implies the existence of a unique factorization of $g$ through $e$.)

2. $\mathscr{A}$ is wellpowered, see [8, Remark 1.56]. This implies that for every object $A$ the poset $\operatorname{Sub}(A)$ of all subobjects of $A$ is a complete lattice.

3. Monomorphisms are closed under filtered colimits (see [8, Proposition 1.62]). We also use the fact (true in every category) that monomorphisms are closed under wide intersections and inverse images.

Since subcoalgebras play a basic role in the whole paper, and quotients are important from Section 3 onwards, we need to make clear what we mean by those. This is the aim of Remark 2.7 and Terminology 2.8.

Remark 2.7. Assuming that $H$ preserves monomorphisms, homomorphisms of coalgebras factorize into those carried by strong epimorphisms followed by those carried by monomorphisms. Moreover, the two classes of homomorphisms form a factorization system in Coalg $H$. Indeed, let $h$ be a coalgebra homomorphism from the coalgebra $(A, \alpha)$ to the coalgebra $(B, \beta)$ and let $h=m \cdot e$ be a (strong epi, mono)-factorization in $\mathscr{A}$, then the diagonal fill-in property yields a coalgebra for which $m$ and $e$ are homomorphisms:

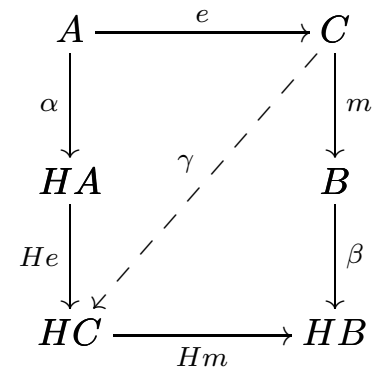

The diagonal fill-in property in Coalg $H$ follows easily, too.

We also point out that the monomorphisms of Coalg $H$ need not be carried by monomorphisms in $\mathscr{A}$.

Terminology 2.8. When we speak about subcoalgebras of a coalgebra $(A, \alpha)$ we mean those represented (up to isomorphism) by homomorphisms $m:\left(A^{\prime}, \alpha^{\prime}\right) \longrightarrow(A, \alpha)$ with $m$ a monomorphism in $\mathscr{A}$. As usual, if $m$ is not invertible, the subcoalgebra is said to be proper. Quotients of $(A, \alpha)$ are represented by homomorphisms with domain $(A, \alpha)$ carried by a strong epimorphism in $\mathscr{A}$; again, properness means they are not invertible.

Definition 2.9. A cartesian subcoalgebra of a coalgebra $(A, \alpha)$ is a subcoalgebra $m$ : $\left(A^{\prime}, \alpha^{\prime}\right) \hookrightarrow(A, \alpha)$ forming a pullback

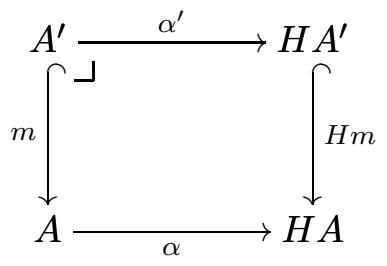

A coalgebra is called well-founded if it has no proper cartesian subcoalgebra. 


\section{Example 2.10.}

(1) The concept of well-founded coalgebra was introduced originally by Osius [22] for the power set functor $\mathscr{P}$. Recall that coalgebras for $\mathscr{P}$ are simply graphs: given $\alpha$ : $A \rightarrow \mathscr{P} A$, then $\alpha(x)$ is the set of neighbors of $A$ in the graph. However, coalgebra homomorphisms $h: A \rightarrow B$ are stronger than graph homomorphisms: $h$ not only preserves edges of $A$, but also for every edge $h(a) \rightarrow b$ in $B$ there exists an edge $a \rightarrow a^{\prime}$ in $A$ with $b=h\left(a^{\prime}\right)$. Then a subcoalgebra of $A$ is an (induced) subgraph $A^{\prime}$ with the property that every neighbor of a vertex of $A^{\prime}$ lies in $A^{\prime}$. The subgraph $A^{\prime}$ is cartesian iff it contains every vertex all of whose neighbors lie in $A^{\prime}$.

The graph $A$ is a well-founded coalgebra iff it has no infinite path. Indeed, the set $A^{\prime}$ of all vertices lying on no infinite path forms clearly a cartesian subcoalgebra. And $A$ is well-founded iff $A=A^{\prime}$.

(2) Let $A$ be a deterministic automaton considered as a coalgebra for $H X=X^{I} \times\{0,1\}$. A subcoalgebra $A^{\prime}$ is cartesian iff it contains every state all whose successors (under the inputs from $I$ ) lie in $A^{\prime}$. This holds, in particular, for $A^{\prime}=\emptyset$. Thus, no nonempty automaton is well-founded.

(3) Coalgebras for $H X=X+1$ are dynamical systems with deadlocks. A subcoalgebra $A^{\prime}$ of a coalgebra $A$ is cartesian iff $A^{\prime}$ contains all deadlocks and every state whose next state lies in $A^{\prime}$. So a dynamical system is well-founded iff it has no infinite computation.

Proposition 2.11. Initial algebras are, as coalgebras, well-founded.

Remark. No assumptions on the base category are needed in the proof.

Proof. Let $\varphi: H I \rightarrow I$ be an initial algebra. Given a pullback

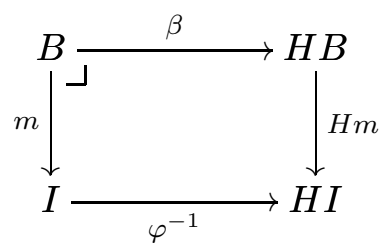

with $m$ monic, we prove that $m$ is invertible. It is clear that $\beta$ is invertible (since $\varphi^{-1}$ is), and for the algebra $\beta^{-1}: H B \rightarrow B$ there exists an algebra homomorphism $f:(I, \varphi) \rightarrow\left(B, \beta^{-1}\right)$. Since $m$ is also an algebra homomorphism, we conclude that $m f$ is an endomorphism of the initial algebra. Thus, $m f=\mathrm{id}$, proving that $m$ is invertible.

Remark 2.12. In contrast, final coalgebras are never well-founded, unless they coincide with initial algebras.

To prove this, we are going to use the initial chain defined in [3]. This is the chain

$$
H^{i} 0 \quad(i \in \text { Ord }) \quad \text { and } \quad w_{i j}: H^{i} 0 \longrightarrow H^{j} 0 \quad(i \leq j)
$$

defined uniquely up to natural isomorphism by

$$
\begin{aligned}
& H^{0} 0=0 \quad \text { (initial object of } \mathscr{A} \text { ) } \\
& H^{i+1} 0=H H^{i} 0 \quad \text { and } \quad w_{i+1, j+1}=H w_{i, j}
\end{aligned}
$$

and for limit ordinals $i$

$$
H^{i} 0=\underset{j<i}{\operatorname{colim}} H^{j} 0 \quad \text { with colimit cocone } w_{i j} \quad(i<j) .
$$


The chain is said to converge at $i$ if the connecting map $w_{i, i+1}: H^{i} 0 \longrightarrow H H^{i} 0$ is invertible. The inverse then makes $H^{i} 0$ an initial algebra.

Proposition 2.13 ([32]). Let $H$ preserve monomorphisms.

(1) Whenever there exists a fixed point of $H$, i.e. an object $X \cong H X$, then $H$ has an initial algebra.

(2) If $H$ has an initial algebra, then the initial chain converges.

Remark 2.14. This result was shown in Theorem II.4 of [32]. The proof uses 2. and 3. of Remark 2.6. It is based on the fact that the isomorphism $u: H X \rightarrow X$ yields a cone $m_{i}: H^{i} 0 \rightarrow X(i \in$ Ord $)$ of the initial chain with all $m_{i}$ monic: $m_{0}: 0 \rightarrow X$ is unique and $m_{i+1}=u \cdot H m_{i}$. Thus, the initial chain converges because it is a chain of subobjects of $X$.

Proposition 2.15. If $H$ preserves monomorphisms, the only well-founded fixed points of $H$ are the initial algebras.

Proof. Let $u: H X \stackrel{\sim}{\rightarrow} X$ be a fixed point such that $u^{-1}: X \rightarrow H X$ is a well-founded coalgebra. Then we prove that $(X, u)$ is an initial algebra. Let $m_{i}: H^{i} 0 \rightarrow X$ be the cone of Remark 2.14. We know that there exists an ordinal $j$ such that $m_{j}$ and $m_{j+1}$ represent the same subobject, thus, $w_{j, j+1}: H^{i} 0 \rightarrow H\left(H^{i} 0\right)$ is invertible. Consequently, $w_{j, j+1}^{-1}: H\left(H^{i} 0\right) \rightarrow H^{i} 0$ is an initial algebra.

The following square

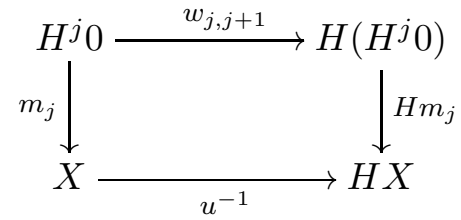

commutes: by definition we have $m_{j+1}=u \cdot H m_{j}$ and since $m_{j}=m_{j+1} \cdot w_{j, j+1}$ (due to the compatibility of the $m_{i}$ 's) we conclude

$$
m_{j}=u \cdot H m_{j} \cdot w_{j, j+1} .
$$

Since both horizontal arrows are invertible, the square above is a pullback. From the well-foundednes of $\left(X, u^{-1}\right)$ we conclude that $m_{j}$ is invertible. Thus, the algebra $(X, u)$ is isomorphic to the initial algebra $\left(H^{j} 0, w_{j, j+1}^{-1}\right)$ via $m_{j}$. This proves that $(X, u)$ is initial.

Corollary 2.16. If $H$ preserves monomorphisms and has a well-founded final coalgebra, then the initial algebra and final coalgebra coincide.

Example 2.17. This demonstrates that the assumption that $H$ preserves monomorphisms is essential. Consider the category Gra of graphs and graph morphisms (i.e., functions preserving edges). All assumptions in 2.3 are fulfilled. The endofunctor

$$
H X= \begin{cases}X+\{t\} \text { (no edges) } & \text { if } X \text { has no edges } \\ 1, \text { terminal graph, } & \text { else. }\end{cases}
$$

does not preserve monomorphisms. Its final coalgebra $1=H 1$ is well-founded because neither of the two proper subcoalgebras is cartesian. However, the initial algebra is carried by an infinite graph without edges. 
Definition 2.18. Assume that $H$ preserves monomorphisms. Then for every coalgebra $\alpha: A \longrightarrow H A$ we denote by $\bigcirc$ the endofunction on $\mathbf{S u b}(A)$ (see Remark 2.6,2) assigning to every subobject $m: A^{\prime} \longrightarrow A$ the inverse image of $H m$ under $\alpha$, i. e., we have a pullback square:

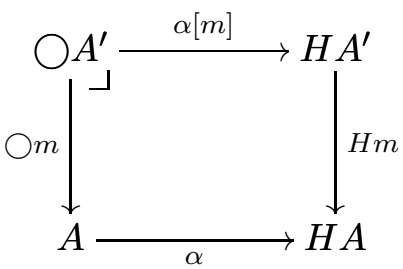

This function $m \longmapsto \bigcirc m$ is obviously order-preserving. By the Knaster-Tarski fixed point theorem, it has a least fixed point.

Corollary 2.19. A coalgebra $(A, a)$ is well-founded iff the least fixed point of $\bigcirc$ is all of A.

Incidentally, the notation $\bigcirc m$ comes from modal logic, especially the areas of temporal logic where one reads $\bigcirc \phi$ as " $\phi$ is true in the next moment," or "next time $\phi$ " for short.

Example 2.20. Recall our discussion of graphs from Example 2.10 (1). The pullback $\bigcirc A^{\prime}$ of a subgraph $A^{\prime}$ is the set of points in the graph $A$ all of whose neighbors belong to $A^{\prime}$.

Remark 2.21. As we mentioned in the introduction, the concept of well-founded coalgebra was introduced by Taylor [28, 29]. Our formulation is a bit simpler. In [29, Definition 6.3.2] he calls a coalgebra $(A, \alpha)$ well-founded if for every pair of monomorphisms $m: U \longrightarrow A$ and $h: H \longrightarrow U$ such that $h \cdot m$ is the inverse image of $H m$ under $\alpha$ it follows that $m$ is an isomorphism. Thus, in lieu of fixed points of $m \longmapsto \bigcirc m$ he uses pre-fixed points.

In addition, our overall work has a methodological difference from Taylor's that is worth mentioning at this point. Taylor is giving a general account of recursion and induction, and so he is concerned with general principles that underlie these phenomena. Indeed, he is interested in settings like non-boolean toposes where classical reasoning is not necessarily valid. On the other hand, in this paper we are studying initial algebras, final coalgebras, and similar concepts, using standard classical mathematical reasoning. In particular, we make free use of transfinite induction.

Notation 2.22.

(a) Assume that $H$ preserves monomorphisms. For every coalgebra $\alpha: A \longrightarrow H A$ denote by

$$
a^{*}: A^{*} \longrightarrow A
$$

the least fixed point of the function $m \longmapsto \bigcirc m$ of Definition 2.18, (Thus, $(A, \alpha)$ is well-founded iff $a^{*}$ is invertible.) Since $a^{*}$ is a fixed point we have a coalgebra structure $\alpha^{*}: A^{*} \longrightarrow H A^{*}$ making $a^{*}$ a coalgebra homomorphism.

(b) For every coalgebra $\alpha: A \longrightarrow H A$ we define a chain of subobjects

$$
a_{i}^{*}: A_{i}^{*} \longrightarrow A \quad(i \in \mathbf{O r d})
$$


of $A$ in $\mathscr{A}$ by transfinite recursion: $a_{0}^{*}: 0 \longrightarrow A$ is unique; given $a_{i}^{*}$, define $a_{i+1}^{*}$ by the pullback

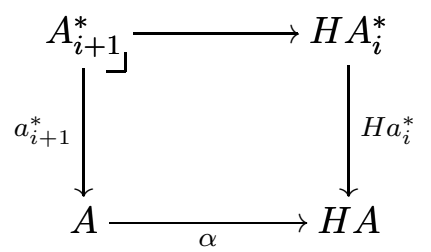

and for limit ordinals $i$ define $a_{i}^{*}: A_{i}^{*} \longrightarrow A$ to be the union of the chain of monomorphisms $a_{j}^{*}: A_{j}^{*} \longrightarrow A$,

$$
a_{i}^{*}=\bigcup_{j<i} a_{j}^{*}
$$

It is easy to prove by transfinite induction that all $a_{i}^{*}$ are monic (for $i=0$ recall that 0 is simple). Moreover, for every limit ordinal $i$ the union above coincides with the colimit of the chain, that is, the monomorphism $a_{i}^{*}: A_{i}^{*} \longrightarrow A$ is just the induced morphism from the colimit of the chain to $A$, see Remark 2.6, point 3 .

Remark 2.23. We observe that for all ordinals $i \leq j$ the connecting maps

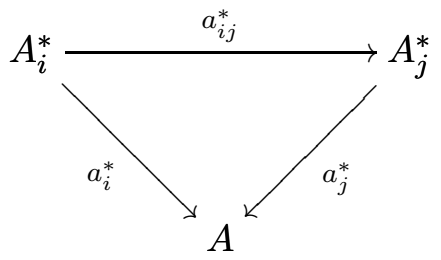

of the chain of Notation 2.22 form the following commutative diagram which can be used as a definition of the maps $a_{i j}^{*}$ (via the universal property of pullbacks):

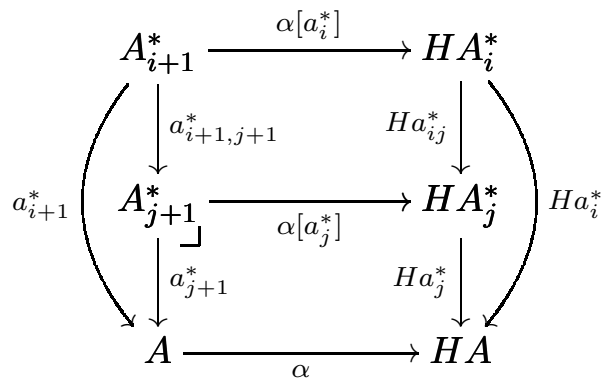

Remark 2.24. This way, what we have is nothing else than the construction of the least fixed point of $m \longmapsto \bigcirc m$, see Remark 2.21, in the proof of the Knaster-Tarski Theorem in [27. Thus, $a^{*}=\bigcup_{i \in \text { Ord }} a_{i}^{*}$. However, since $A$ has only a set of subobjects,

$$
a^{*}=a_{i_{0}}^{*} \quad \text { for some ordinal } i_{0} \text {. }
$$

And for this ordinal $i_{0}$, an easy verification shows that the coalgebra structure of $A^{*}$ above is

$$
\alpha^{*}=\alpha\left[a_{i_{0}}^{*}\right]=\alpha\left[a^{*}\right]
$$

Henceforth, we call $A^{*}$ the smallest cartesian subcoalgebra of $A$.

From now on, whenever we use the notations $\bigcirc m$ and $a^{*}$, we only do so when $H$ preserves monomorphisms. 
Example 2.25. On the category Gra consider the functor $H$ of Example 2.17, It has $1=H 1$ as its final coalgebra, and this coalgebra is well-founded. However, for $\alpha$ as id : 1 $\longrightarrow H 1$ (the final coalgebra), there is no ordinal $i$ such that $a_{i}^{*}=\mathrm{id}_{1}$. This shows that Notation 2.22 is meaningful only if we assume that $H$ preserves monomorphisms.

Example 2.26. For every graph $A$ considered as a coalgebra for $\mathscr{P}, A^{*}$ is the subgraph on all vertices of $A$ from which no infinite path starts. Since $m \mapsto \bigcirc m$ is not necessarily continuous, the ordinal $i_{0}$ of (2.5) above can be arbitrarily large. Here is an example with $i_{0}=\omega+1$ :

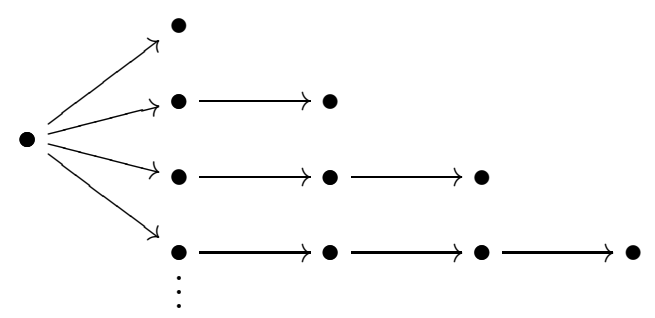

Proposition 2.27. If $H$ preserves monomorphisms then well-founded coalgebras form a full coreflective subcategory of Coalg $H$ : For every coalgebra $(A, \alpha)$, the smallest cartesian subcoalgebra $\left(A^{*}, \alpha^{*}\right)$ is its coreflection.

Remark. We thus prove that $\left(A^{*}, \alpha^{*}\right)$ is well-founded, and for every homomorphism $f:(B, \beta) \longrightarrow(A, \alpha)$ with $(B, \beta)$ well-founded there exists a unique homomorphism

$$
\bar{f}:(B, \beta) \longrightarrow\left(A^{*}, \alpha^{*}\right) \quad \text { with } \quad f=a^{*} \cdot \bar{f} .
$$

Proof. (i) $\left(A^{*}, \alpha^{*}\right)$ is clearly well-founded: From Definition 2.18 and Notation 2.22, we know that $\left(A^{*}, a^{*}\right)$ is the least fixed point of $\bigcirc: \mathbf{S u b}(A) \longrightarrow \mathbf{S u b}(A)$, that is, $\left(A^{*}, \alpha^{*}\right)$ is the smallest cartesian subcoalgebra of $(A, \alpha)$. Then $\left(A^{*}, \alpha^{*}\right)$ cannot have proper cartesian subcoalgebras since its cartesian subcoalgebras are cartesian subcoalgebras of $(A, \alpha)$.

(ii) Since $a^{*}$ is a monomorphism there is at most one coalgebra homomorphism $\bar{f}: B$ $\longrightarrow A^{*}$ with $a^{*} \cdot \bar{f}=f$. Thus, we are finished if we show that $\bar{f}$ exists. To this end, for all ordinals $i \leq j$, let $a_{i j}^{*}: A_{i}^{*} \longrightarrow A_{j}^{*}$ be the connecting maps of the chain of Remark 2.23. Analogously, use $b_{i j}^{*}: B_{i}^{*} \longrightarrow B_{j}^{*}$ for the chain of the subobjects $b_{i}^{*}: B_{i}^{*} \longrightarrow B$, whose union is $B^{*}=B$. We define the components of a natural transformation $\bar{f}_{i}: B_{i}^{*} \longrightarrow A_{i}^{*}$, $i \in$ Ord, by transfinite recursion on ordinals $i$, satisfying

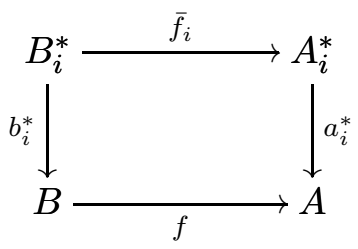


Let $\bar{f}_{0}=\mathrm{id}: 0 \longrightarrow 0$. For isolated steps consider the diagram below:

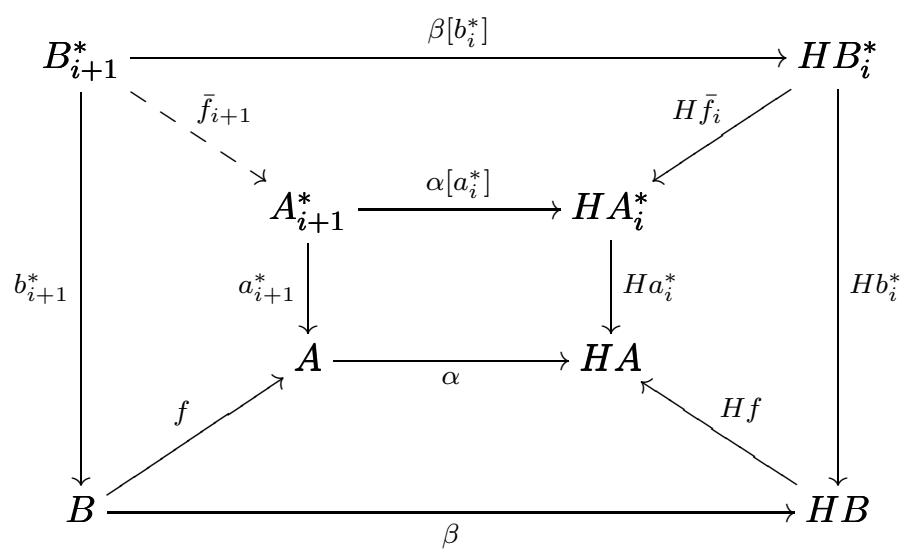

The inner and outside squares commute by the definition of $A_{i+1}^{*}$ and $B_{i+1}^{*}$, respectively. For the lower square we use that $f$ is a coalgebra homomorphism, and the right-hand one commutes by the induction hypothesis. The inner pullback induces the desired morphism $\bar{f}_{i+1}$ and the commutativity of the left-hand square is that of (2.7) for $i+1$. Finally, for a limit ordinal $j$ let $\bar{f}_{j}=\operatorname{colim}_{i<j} \bar{f}_{i}$, in other words, $\bar{f}_{j}$ is the unique morphism such that the squares

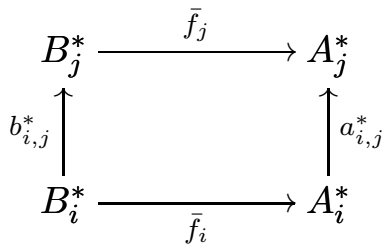

commute for all $i<j$. It is easy to prove by transfinite induction that $\bar{f}_{j}: B_{j}^{*} \rightarrow A_{j}^{*}$ is natural in $j$.

We need to verify that (2.7) commutes for $\bar{f}_{j}$. This is clear for $j=0$ and for $j$ isolated this follows from the definition of $\bar{f}_{i+1}$. Let $j$ be a limit ordinal. Then (2.7) commutes due to the following diagram for every $i<j$ :

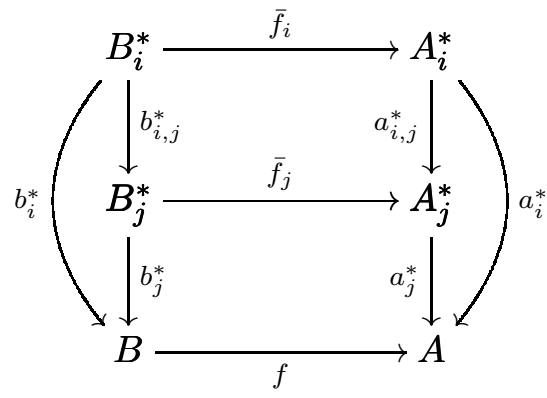

To complete the proof consider any ordinal $i$ such that $B_{i}^{*}=B^{*}=B$ and $A_{i}^{*}=A^{*}$ hold. Then $\bar{f}=\bar{f}_{i}: B \longrightarrow A^{*}$ is a coalgebra homomorphism with $a_{i}^{*} \cdot \bar{f}=f$ by the commutativity of the upper and left-hand parts of Diagram (2.8). 
For endofunctors preserving inverse images the following corollary is Exercise VI.16 in [29]:

Corollary 2.28. Assuming that $H$ preserves monomorphisms, the subcategory of Coalg $H$ consisting of the well-founded coalgebras is closed under quotients and coproducts in Coalg $H$.

This follows from a general result on coreflective subcategories: the category Coalg $H$ has a (strong epi, mono)-factorization system (see Remark 2.6), and its full subcategory of well-founded coalgebras is coreflective with monomorphic coreflections (see Proposition 2.27). Consequently, it is closed under quotients and colimits.

We also have the following fact which will be used in Section 3.

Lemma 2.29. If $H$ preserves finite intersections, then every subcoalgebra of a well-founded coalgebra is well-founded.

Proof. Given a subcoalgebra $f:(B, \beta) \longrightarrow(A, \alpha)$ we prove that the natural transformation $\bar{f}_{i}: B_{i}^{*} \longrightarrow A_{i}^{*}$ of (2.9) makes the squares in (2.7) pullbacks for every ordinal number $i$. The base case $i=0$ is clear. For the isolated step we use that $a_{i+1}^{*}: A_{i+1}^{*} \longrightarrow A$ is the pullback of $H a_{i}^{*}$ along $\alpha$. Thus, it suffices to show that $b_{i+1}^{*}: B_{i+1} \longrightarrow B$ is a pullback of $H a_{i}^{*}$ along $\alpha \cdot f$. But, since $\alpha \cdot f=H f \cdot \beta$ and since $H$ preserves finite intersections (i.e., pullbacks of monos along monos), the latter pullback can be obtained by pasting two pullback squares as displayed below:

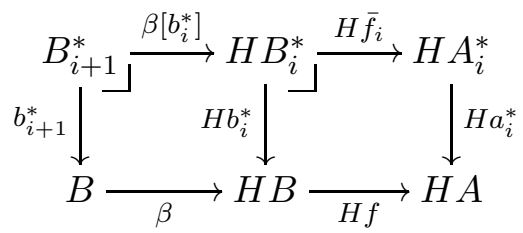

Now assume that $(A, \alpha)$ is well-founded, i. e., some $a_{i}^{*}$ is invertible. Then its pullback $b_{i}^{*}$ along $f$ is invertible, i. e., $(B, \beta)$ is well-founded.

Remark 2.30. If $H$ is a set functor which also preserves inverse images, a much stronger result holds, as proved in [29, Corollary 6.3.6]: every coalgebra from which a homomorphism into a well-founded coalgebra exists is well-founded.

Example 2.31. Without the assumption that $H$ preserves finite intersections the lemma above can fail to be true. On the category Gra of graphs the functor $H$ of Example 2.17 has the well-founded coalgebra $1=H 1$ which has the subcoalgebra

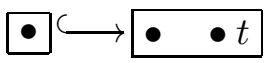

which is not well-founded: its subcoalgebra $\emptyset \longrightarrow\{t\}$ is cartesian.

\subsection{Recursive coalgebras.}

Here we recall the notion of recursive coalgebra in order to use it for our proof that initial algebras are the same as final well-founded coalgebras. "Recursive" and "well-founded" are closely related concepts. But whereas final recursive coalgebras are already known to be initial algebras, see [12, for well-founded coalgebras this is new (and a bit more involved). 
Definition 2.32. A coalgebra $\alpha: A \longrightarrow H A$ is recursive if for every algebra $\beta: H B$ $\longrightarrow B$ there exists a unique coalgebra-to-algebra homomorphism

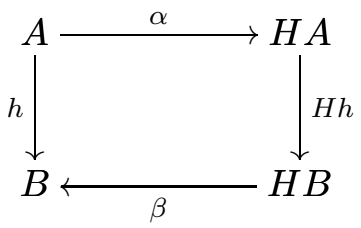

This concept was introduced by Taylor under the name "coalgebra obeying the recursion scheme", the name recursive coalgebra stems from Capretta et al. [12].

Examples 2.33 (see [12]).

(1) $0 \longrightarrow H 0$ is a recursive coalgebra.

(2) If $\alpha: A \longrightarrow H A$ is recursive, then so is $H \alpha: H A \longrightarrow H H A$.

(3) A colimit of recursive coalgebras is recursive. Combining these results we see that in the initial chain (2.1) all the coalgebras

$$
w_{i, i+1}: H^{i} 0 \longrightarrow H H^{i} 0
$$

are recursive.

We are going to prove that for set functors, well-founded coalgebras are recursive. Before we do this, let us discuss the converse. In general, recursive coalgebras need not be well-founded, even for set functors. However for all set functors preserving inverse images recursiveness is equivalent to well-foundedness, as shown by Taylor [28, 29].

Example 2.34 (see [5]). A recursive coalgebra need not be well-founded. Let $H$ : Set $\longrightarrow$ Set be defined on objects by

$$
H X=\left(X \times X \backslash \Delta_{X}\right)+\{d\}
$$

where $\Delta_{X}$ denotes the diagonal of $X$. For morphisms $f: X \longrightarrow Y$ we take $H f(d)=d$ and

$$
H f\left(x_{1}, x_{2}\right)= \begin{cases}d & \text { if } f\left(x_{1}\right)=f\left(x_{2}\right) \\ \left(f x_{1}, f x_{2}\right) & \text { else }\end{cases}
$$

This functor $H$ preserves monomorphisms. The coalgebra $A=\{0,1\}$ with the structure $\alpha$ constant to $(0,1)$ is recursive: given an algebra $\beta: H B \longrightarrow B$, the unique coalgebra-toalgebra homomorphism $h:\{0,1\} \longrightarrow B$ is

$$
h(0)=h(1)=\beta(d)
$$

But $A$ is not well-founded: $\emptyset$ is a cartesian subcoalgebra.

Theorem 2.35. If $H$ preserves monomorphisms, then every well-founded coalgebra is recursive.

For functors preserving inverse images this follows from [28, Theorem 6.3.13].

Proof. Let $\alpha: A \longrightarrow H A$ be well-founded. For every algebra $e: H X \longrightarrow X$ we prove the existence and uniqueness of a coalgebra-to-algebra homomorphism $A \longrightarrow X$. We use the initial chain $\left(H^{i} 0\right)$ of (2.1) and also the chain $\left(A_{i}^{*}\right)$ from Notation 2.22.

(1) Existence. We prove first that there is a unique natural transformation

$$
f_{i}: A_{i}^{*} \longrightarrow H^{i} 0 \quad(i \in \mathbf{O r d})
$$


such that for all ordinals $i$ we have

$$
f_{i+1}=\left(A_{i+1}^{*} \stackrel{\alpha\left[a_{i}^{*}\right]}{\longrightarrow} H A_{i}^{*} \stackrel{H f_{i}}{\longrightarrow} H\left(H^{i} 0\right)=H^{i+1} 0\right) .
$$

In fact, since both of the transfinite chains $\left(A_{i}^{*}\right)$ and $\left(H^{i} 0\right)$ are defined by colimits on all limit ordinals $i$, and $f_{0} \operatorname{must}_{\text {be }} \mathrm{id}_{\emptyset}$, we only need to check the commutativity of the square

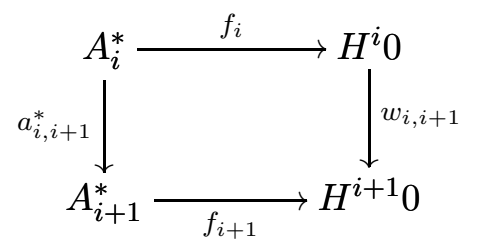

for every successor ordinal $i$. For this, the diagram below commutes by the induction hypothesis (2.12) and by the commutativity of the upper inner square of (2.4) in Remark 2.23 ,

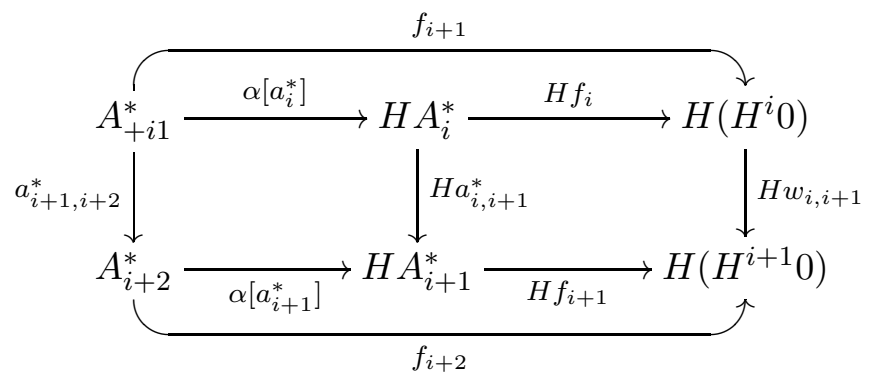

Next, since the $H^{i} 0$ are recursive coalgebras (see Example 2.33) we have unique coalgebra-to-algebra homomorphisms into $X$. These form a natural transformation into the constant functor with value $X$ :

$$
r_{i}: H^{i} 0 \longrightarrow X \quad(i \in \text { Ord })
$$

Consequently, we obtain a natural transformation $r_{i} f_{i}: A_{i}^{*} \longrightarrow X$ which, for $i$ such that $A_{i}^{*}=A_{i+1}^{*}$ (thus, $\left.A=A_{i}^{*}\right)$, yields

$$
h=r_{i} f_{i}: A \longrightarrow X .
$$

Now consider the diagram below:

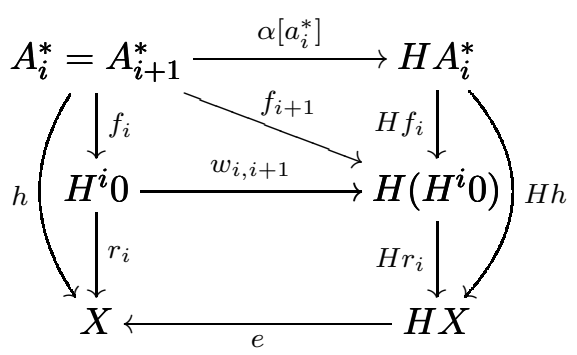

The morphism at the top is $\alpha^{*}$, by (2.6). The sides are the definition of $h$, the bottom square is the definition of $r_{i}$, and the upper right-hand triangle is the definition of $f_{i+1}$. The upper left-hand triangle is (2.12) since $a_{i, i+1}^{*}=\mathrm{id}$. The overall outside of the figure shows that $h$ is a coalgebra-to-algebra homomorphism as desired. 
(2) Uniqueness. If $h_{1}, h_{2}: A \longrightarrow X$ are coalgebra-to-algebra homomorphisms, then we prove $h_{1}=h_{2}$ by showing that

$$
h_{1} \cdot a_{i}^{*}=h_{2} \cdot a_{i}^{*} \quad \text { for all } i \in \text { Ord. }
$$

The case $i=0$ is clear, in the isolated step use the commutative diagrams (with $t=1,2$ ):

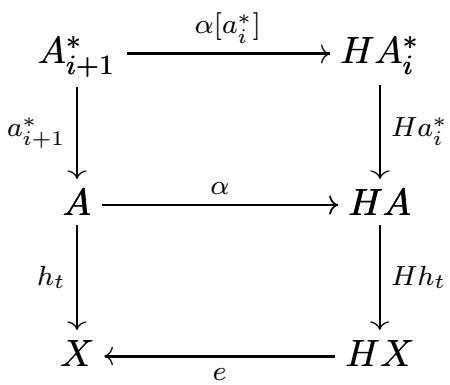

and the limit steps follow from $A_{j}^{*}=\operatorname{colim}_{i<j} A_{i}^{*}$ for limit ordinals $j$.

Example 2.36. There is a $\mathscr{P}$-algebra $(B, \beta)$ such that for all $\mathscr{P}$-coalgebras $(A, \alpha)$, if $(A, \alpha)$ is not well-founded, then there are at least two coalgebra-to-algebra homomorphisms $h: A$ $B$.

We take $B=\{0,1,2\}$, with $\beta: \mathscr{P} B \longrightarrow B$ defined as follows:

$$
\beta(x)= \begin{cases}0 & \text { if } x=\emptyset \text { or } x=\{0\} \\ 1 & \text { else if } 1 \in x \\ 2 & \text { if } 2 \in x \text { and } 1 \notin x\end{cases}
$$

If $(A, \alpha)$ is any coalgebra which is not well-founded, we show that there are at least two coalgebra-to-algebra homomorphisms $h: A \longrightarrow B$. We can take

$$
h_{1}(x)= \begin{cases}0 & \text { if there are no infinite sequences } x=x_{0} \rightarrow x_{1} \rightarrow x_{2} \cdots \\ 1 & \text { if there is an infinite sequence } x=x_{0} \rightarrow x_{1} \rightarrow x_{2} \cdots\end{cases}
$$

and also $h_{2}$ defined the same way, but using 2 as a value instead of 1 . The verification that $h_{1}$ and $h_{2}$ are coalgebra-to-algebra homomorphisms hinges on two facts: first, $h(x)=0$ iff there is no infinite sequence starting from $x$; and second, if $h_{i}(x) \neq 0$, then there is some $y \in \alpha(x)$ such that $h_{i}(y) \neq 0$ as well.

For endofunctors preserving inverse images the following theorem is Corollary 9.9 of [28]. As we mentioned in the introduction, it is non-trivial to relax the assumption on the endofunctor, and so our proof is different from Taylor's. As a result we obtain in Theorem 2.46 below that for a set endofunctor no assumptions are needed.

Remark 2.37. The concepts "initial algebra" and "final recursive coalgebra" coincide for all endofunctors, as proved by Capretta et al. [12. This is not true in general for wellfoundedness in lieu of recursiveness, see Example 2.39 below. But it is true if $H$ preserves finite intersections:

Theorem 2.38. If $H$ preserves finite intersections, then

$$
\text { initial algebra }=\text { final well-founded coalgebra }
$$

That is, an algebra $\varphi: H I \longrightarrow I$ is initial iff $\varphi^{-1}: I \longrightarrow H I$ is the final well-founded coalgebra. 
Proof. Recall that since $H$ preserves finite intersections, i.e., pullbacks of monomorphisms, it preserves monomorphisms (since $m$ is monic iff the pullback of $m$ along itself is formed by identity morphisms).

(a) Let $I$ be an initial algebra. By Remark 2.37, $I$ is a final recursive coalgebra. Also, $I$ is well-founded by Proposition 2.11, Thus by Theorem 2.35, it is a final well-founded coalgebra.

(b) Let $\psi: I \longrightarrow H I$ be a final well-founded coalgebra.

(b1) Factorize $\psi=m \cdot e$ where $e$ is a strong epimorphism and $m$ a monomorphism (Remark 2.6). By diagonal fill-in

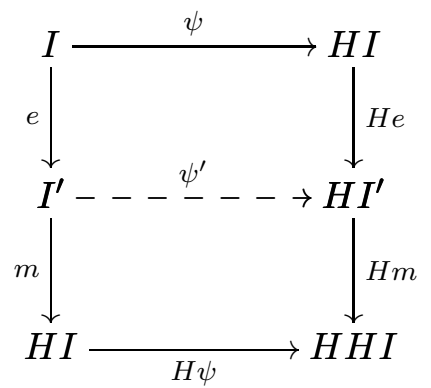

we obtain a quotient $\left(I^{\prime}, \psi^{\prime}\right)$ which, by Corollary 2.28, is well-founded. Consequently, a coalgebra homomorphism $f:\left(I^{\prime}, \psi^{\prime}\right) \longrightarrow(I, \psi)$ exists. Then $f e$ is an endomorphism of the final well-founded coalgebra, hence, $f e=\operatorname{id}_{I}$. This proves that $e$ is an isomorphism, in other words

$$
\psi \text { is a monomorphism. }
$$

(b2) The coalgebra $(H I, H \psi)$ is well-founded. Indeed, consider a cartesian subcoalgebra $\left(A^{\prime}, a^{\prime}\right)$

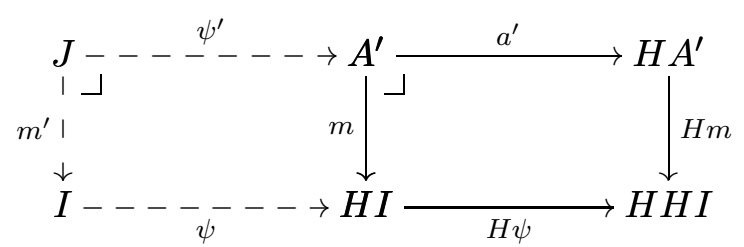

Form the intersection $J$ of $m$ and $\psi$. Since $H$ preserves this intersection, it follows that $m$ and $H m^{\prime}$ represent the same subobject of $H I$, thus, we have

$$
u: A^{\prime} \longrightarrow H J, \quad \text { with } \quad m=H m^{\prime} \cdot u \text {. }
$$

This yields a cartesian subcoalgebra

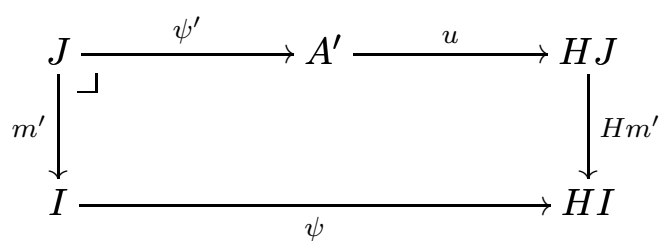

and since $(I, \psi)$ is well-founded, we conclude that $m^{\prime}$ is invertible. Consequently, $m=H m^{\prime} \cdot u$ is invertible. 
(b3) $\psi$ is invertible. Indeed, we have, by (b2), a homomorphism $h:(H I, H \psi) \longrightarrow$ $(I, \psi)$ :

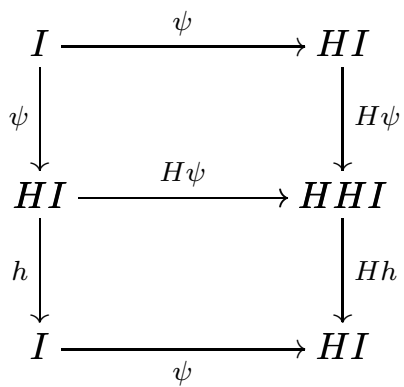

Then $h \cdot \psi$ is an endomorphism of $(I, \psi)$, thus, $h \cdot \psi=$ id. And the lower square yields $\psi \cdot h=H(h \cdot \psi)=\mathrm{id}$, whence $I \cong H I$,

(b4) By Proposition 2.13, the initial chain converges, and $w_{i, i+1}^{-1}: H H^{i} 0 \longrightarrow H^{i} 0$ is an initial algebra for some ordinal $i$. Moreover, $w_{i, i+1}: H^{i} 0 \longrightarrow H H^{i} 0$ is by (a) a final well-founded coalgebra, thus, isomorphic to $\psi: I \longrightarrow H I$. Therefore $\left(I, \psi^{-1}\right)$ is isomorphic to the initial algebra above.

\section{Example 2.39.}

(a) For the identity functor on the category of rings the initial algebra is $\mathbb{Z}$ and the terminal well-founded coalgebra is 1 . This shows the importance of our assumption that the base category have a simple initial object.

(b) Also the assumption that $H$ preserves finite intersections is important: The endofunctor $H$ of Gra in Example2.17 has 1 as its final well-founded coalgebra, and its initial algebra is infinite.

Remark 2.40. Although we have previously worked with monomorphisms only, the whole theory can be developed for a general class $\mathscr{M}$ of monomorphisms in the base category $\mathscr{A}$. We need to assume that

(a) $\mathscr{A}$ is $\mathscr{M}$-wellpowered,

(b) $\mathscr{M}$ is closed under inverse images, and

(c) $\mathscr{M}$ is constructive in the sense of [32].

The last point means that $\mathscr{M}$ is closed under composition, and for every chain of monomorphisms in $\mathscr{M}$, (i) a colimit exists and is formed by monomorphisms in $\mathscr{M}$, and (ii) the factorization morphism of every cocone of monomorphisms in $\mathscr{M}$ is again a monomorphism in $\mathscr{M}$. This in particular states that the initial object has the property that all morphisms $0 \rightarrow X$ lie in $\mathscr{M}$.

We then can define $\mathscr{M}$-well-founded coalgebra as one that has no proper cartesian subcoalgebra carried by an $\mathscr{M}$-monomorphism.

All results above hold in this generality. In Theorem 2.35 we must assume that $H$ preserves $\mathscr{M}$, that is, if $m$ lies in $\mathscr{M}$ then so does $H m$. In Theorem 2.38 we need to assume that $H$ preserves $\mathscr{M}$ and finite intersections of $\mathscr{M}$-monomorphisms.

Example 2.41. All LFP categories with simple initial object satisfy all the assumptions of 2.3 for

$$
\mathscr{M}=\text { strong monomorphisms, }
$$

see [8, Propositions 1.61 and 1.62]. 
Example 2.42. Here we compare well-foundedness w.r.t to monomorpisms to that w.r.t. strong monomorphisms. Take again the category Gra of graphs and graph morphisms and $H$ be the following endofunctor: The nodes of $H A$ are all finite independent sets $a \subseteq A$ (i.e., no edge lies in $a$ ) plus a new node $t$. The coalgebra structure is the constant map to $\{t\}$, i.e., the only edges of $H A$ connect every node to $t$ ( $t$ is a loop). For a graph morphism $f: A \longrightarrow B$, we take $H f: H A \longrightarrow H B$ to be

$$
H f(a)= \begin{cases}f[a] & \text { if } f[a] \text { is independent in } B \\ t & \text { otherwise }\end{cases}
$$

This functor clearly preserves strong monomorphisms (but not monomorphisms).

By Theorem 2.38, the initial algebra for $H$ is the same as its final $\mathscr{M}$-well-founded coalgebra. This is

$$
I=I_{0} \cup\{t\}
$$

where $I_{0}=\mathscr{P}_{f} I_{0}$ is the initial algebra of the finite power set functor on Set, taken as a discrete graph, and the coalgebra structure is the constant to $\{t\}$.

In contrast, $I$ is not well-founded (w. r. t. all monomorphisms). Here is the reason. Let $J$ be the same as $I$, except that we drop all edges between $t$ and the elements of $I_{0}$. (We keep just the loop at $t$.) Then $H J=H I=I$. The inclusion $i: J \longrightarrow I$ is of course monic, and $H i=\mathrm{id}_{H I}$. It is easy to check that this inclusion is a coalgebra morphism, and indeed this subcoalgebra is clearly cartesian. This verifies that $I$ is not well-founded.

\subsection{Initial algebras of set functors.}

The main result of this section is that for all endofunctors $H$ of Set the equality

$$
\text { initial algebra }=\text { final well-founded coalgebra }
$$

holds, i. e., for the particular case of our given LFP category being $\mathscr{A}=$ Set one can lift the assumption that $H$ preserves finite intersections in Theorem 2.38 ,

Proposition 2.43 (Trnková [31]). For every endofunctor $H$ of Set there exists an endofunctor $\bar{H}$ preserving finite intersections and identical with $H$ on all nonempty sets (and nonempty functions).

Remark 2.44. The functor $\bar{H}$ is unique up to natural isomorphism. We call it the Trnková closure of $H$. Let us recall how Trnková defined $\bar{H}$ :

Denote by $C_{01}$ the set functor $\emptyset \longmapsto \emptyset$ and $X \longmapsto 1$ for all $X \neq \emptyset$. Define $\bar{H}$ as $H$ on all nonempty sets, and put

$$
\bar{H} \emptyset=\left\{\tau ; \tau: C_{01} \longrightarrow H \text { a natural transformation }\right\} .
$$

(To check that we have a set here and not a proper class, note that each $\tau: C_{0,1} \longrightarrow H$ is determined by $\tau_{1}: 1 \longrightarrow H 1$. For a nonempty set $A$, if $k: 1 \longrightarrow A$ is arbitrary, $\tau_{A}=H k \circ \tau_{1}$.) Given a nonempty set $X, \bar{H}$ assigns to the empty map $q_{X}: \emptyset \longrightarrow X$ the map

$$
\bar{H} q_{X}: \tau \longmapsto \tau_{X} \quad \text { for every } \quad \tau: C_{01} \longrightarrow H,
$$

where $\tau_{X}: 1 \longrightarrow H X$ is simply an element of $H X$. 
Observe that there exists a map $u: H \emptyset \longrightarrow \bar{H} \emptyset$ such that for every set $A \neq \emptyset$ the triangle

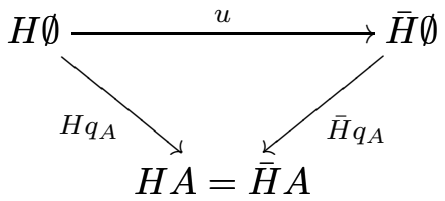

commutes. Indeed, for each element $x \in H \emptyset$, let the natural transformation $u(x): C_{01}$ $\longrightarrow H$ have components $u(x)_{A}=H q_{A}(x)$ for all $A \neq \emptyset$. Then

$$
\bar{H} q_{A}(u(x))=(u(x))_{A}=H q_{A}(x) .
$$

Lemma 2.45. Let $(A, a)$ be a well-founded $H$-coalgebra with $A \neq \emptyset$, so that $(A, a)$ is also an $\bar{H}$-coalgebra. Then $\emptyset$ is not the carrier of any cartesian $\bar{H}$-subcoalgebra of $(A, a)$.

Proof. Assume towards a contradiction that $q_{\bar{H} \emptyset}: \emptyset \longrightarrow \bar{H} \emptyset$ were a cartesian subcoalgebra of $(A, a)$. We claim that the square below is a pullback:

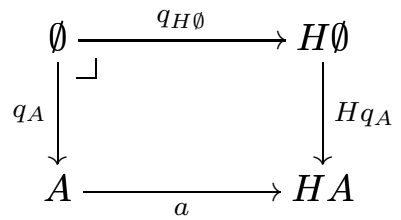

We show that there are no $y \in A$ and $x \in H \emptyset$ such that that $a(y)=H q_{A}(x)$. For assume that $y$ and $x$ exist with these properties. Then by (2.14), $\bar{H} q_{A}(u(x))=a(y)$. This contradicts our assumption that $\left(\emptyset, q_{\bar{H} \emptyset}\right)$ is a cartesian subcoalgebra of $(A, a)$. Thus, $y$ and $x$ do not exist as assumed, and hence, the square in (2.15) is indeed a pullback. Therefore $q_{A}$ is an isomorphism. But $A \neq \emptyset$, and this is a contradiction.

Theorem 2.46. For every endofunctor on Set we have:

$$
\text { initial algebra }=\text { final well-founded coalgebra. }
$$

Proof. Given $H$, we know from Theorem 2.38 that the statement holds for the Trnková closure $\bar{H}$. From this we are going to prove it for $H$.

(a) If $\varphi: H I \longrightarrow I$ is an initial algebra, we prove that $\varphi^{-1}: I \longrightarrow H I$ is a final well-founded coalgebra.

This is clear when $H \emptyset=\emptyset$. In this case $I=\emptyset$. And the only (hence, the final) wellfounded coalgebra is the empty one: if $a: A \longrightarrow H A$ is well-founded, then the following cartesian subcoalgebra

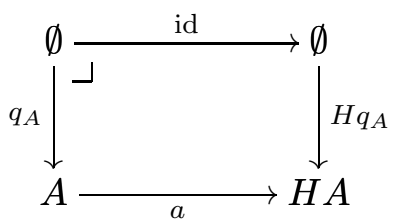

demonstrates that $q_{A}$ is an isomorphism, so $A=\emptyset$.

Thus we assume $H \emptyset \neq \emptyset$. Then $\bar{H} \emptyset \neq \emptyset$ via $u$ in (2.14) above. The $\bar{H}$-algebra $\varphi: \bar{H} I$ $\longrightarrow I$ is initial because every $\bar{H}$-algebra is nonempty, hence, it also is an $H$-algebra. And the unique homomorphism from $I$ w.r.t. $H$ is also a homomorphism w.r.t. $\bar{H}$. By 
Theorem 2.38, $\varphi^{-1}: I \longrightarrow \bar{H} I$ is a final well-founded $\bar{H}$-coalgebra. Let us now verify that it is also well-founded w.r.t. $H$. Consider a cartesian subcoalgebra

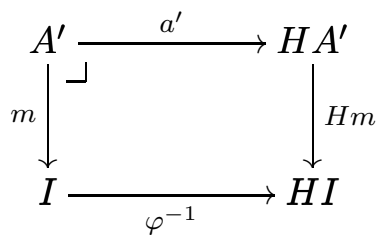

We claim that $A^{\prime}$ cannot be empty. For if it were, then since $H A^{\prime}=H \emptyset \neq \emptyset$, we take any $x \in H A^{\prime}$ and consider $x$ and $(\varphi \cdot H m)(x)$. By the pullback property, there is some $y \in A^{\prime}$ so that $a^{\prime}(y)=x$. This contradicts $A^{\prime}=\emptyset$.

As a result, $H A^{\prime}=\bar{H} A^{\prime}$, and $H m=\bar{H} m$. So (2.17) is a cartesian subcoalgebra for $\bar{H}$. Thus $m$ is invertible, as desired.

At this point we know that $\varphi^{-1}: I \longrightarrow H I$ is a well-founded $H$-coalgebra; we conclude with the verification that $\varphi^{-1}$ is final among these. This follows from the observation that every nonempty well-founded $H$-coalgebra $a: A \longrightarrow H A$ is also well-founded w.r.t. $\bar{H}$. Indeed, consider a cartesian subcoalgebra

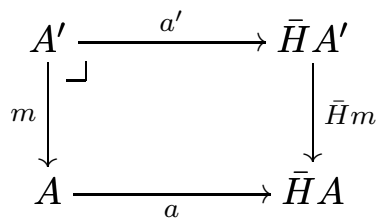

By Lemma 2.45, $A^{\prime} \neq \emptyset$. Thus $\bar{H} m=H m$ and we conclude that $m$ is invertible.

(b) If $\psi: I \longrightarrow H I$ is a final well-founded coalgebra, we prove that $\psi$ is invertible and $\psi^{-1}: H I \longrightarrow I$ is an initial algebra. Unfortunately, we cannot use the converse implication of what we have just proved (every nonempty well-founded $\bar{H}$-coalgebra is also well-founded w.r.t. $H$ ) since this is false in general (see Example 2.47 below). We can assume $H \emptyset \neq \emptyset$, since the case $H \emptyset=\emptyset$ is trivial.

Consider first the coalgebra

$$
b: \bar{H} \emptyset \longrightarrow H \bar{H} \emptyset
$$

defined by

$$
b(\tau)=\tau_{\bar{H} \emptyset} \quad \text { for all } \tau: C_{01} \longrightarrow H .
$$

Let us show that this coalgebra is well-founded for $H$. Consider a cartesian subcoalgebra

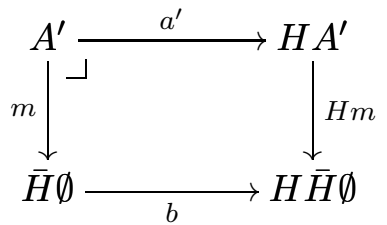

It is our task to prove that $m$ is surjective (thus, invertible). First, assume that $A^{\prime} \neq \emptyset$. Given $\tau: C_{01} \longrightarrow H$ in $\bar{H} \emptyset$, the element $\tau_{A^{\prime}}$ of $H A^{\prime}$ fulfils

$$
b(\tau)=\tau_{\bar{H} \emptyset}=H m\left(\tau_{A^{\prime}}\right)
$$

by the naturality of $\tau$ and the fact that $C_{01} m=\mathrm{id}_{1}$. Thus, there exists an element of $A^{\prime}$ that $m$ maps to $\tau$. Our second case is when $A^{\prime}=\emptyset$. We show that this case leads to a 
contradiction. Observe that $m=q_{\bar{H} \emptyset}: \emptyset \longrightarrow \bar{H} \emptyset$, and let $x \in H \emptyset$, so that $u(x) \in \bar{H} \emptyset$, see (2.14), and we have

$$
b(u(x))=(u(x))_{\bar{H} \emptyset}=H q_{\bar{H} \emptyset}(x) .
$$

Thus $x$ and $u(x)$ are mapped to the same element of $H \bar{H} \emptyset$ by $H m$ and $b$, respectively, contradicting the assumption that $\emptyset$ is a pullback in (2.19) above.

The first point of this coalgebra $(\bar{H} \emptyset, b)$ is that its well-foundedness and non-emptiness implies that the final well-founded $H$-coalgebra $(I, \psi)$ must also be nonempty. Thus $(I, \psi)$ is also a coalgebra for $\bar{H}$. Let us prove that it is well-founded w.r.t. $\bar{H}$. Given a cartesian subcoalgebra

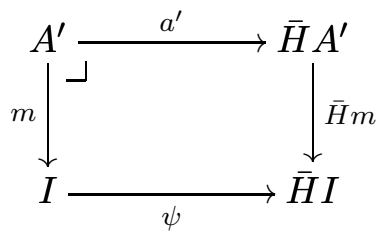

by Lemma 2.45, $A^{\prime} \neq \emptyset$. So $\bar{H} m=H m$, hence $m$ is invertible.

We next prove that $(I, \psi)$ is the final well-founded $\bar{H}$-coalgebra. Let $a: A \longrightarrow \bar{H} A$ be a nonempty well-founded $\bar{H}$-coalgebra. We prove that the coproduct

$$
(A, a)+(\bar{H} \emptyset, b) \quad \text { in Coalg } H
$$

is a well-founded $H$-coalgebra. This will conclude the proof: we have a unique homomorphism from that coproduct into $(I, \psi)$ in Coalg $H$, hence, a unique homomorphism from $(A, a)$ to $(I, \psi)$. Now in oder to prove that the coproduct above is a well-founded $H$ coalgebra we first use that every nonempty well-founded coalgebra for $H$ is also well-founded for $\bar{H}$, thus, both of the summands above are well-founded $\bar{H}$-coalgebras. Since coproducts of coalgebras are formed on the level of sets, the two categories Coalg $H$ and Coalg $\bar{H}$ have the same formation of coproduct of nonempty coalgebras. Let

$$
(A, a)+(\bar{H} \emptyset, b)=(A+\bar{H} \emptyset, c)
$$

be a coproduct in Coalg $\bar{H}$, then this coalgebra is well-founded w.r.t. $\bar{H}$ by Corollary 2.28 , To prove that it is also well-founded w.r.t. $H$, we only need to consider the empty subcoalgebra: we must prove that the square

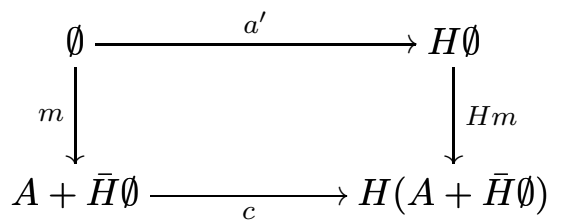

is not a pullback. Indeed, choose an element $x \in H \emptyset$ and put $\tau=u(x)$ (see (2.14)). Then $m=q_{A+\bar{H} \emptyset}$ implies

$$
H m(x)=\tau_{A+\bar{H} \emptyset} .
$$

We also have $\tau \in \bar{H} \emptyset$ and the coproduct injection $v: \bar{H} \emptyset \longrightarrow A+\bar{H} \emptyset$ fulfils $c \cdot v=H v \cdot b$ (due to the formation of coproducts in Coalg $\bar{H}$ ). Therefore

$$
c(v(\tau))=H v(b(\tau))=H v\left(\tau_{\bar{H} \emptyset}\right)=\tau_{A+\bar{H} \emptyset}=H m(x) .
$$

Since we presented elements of $A+\bar{H} \emptyset$ and $H \emptyset$ that are mapped to the same element by $c$ and $H m$, respectively, the square above is not a pullback. This finishes the proof that $(I, \psi)$ is a final well-founded $\bar{H}$-coalgebra. 
By Theorem 2.38 we conclude that $\psi$ is invertible and $\left(I, \psi^{-1}\right)$ is an initial $\bar{H}$-algebra. It is also an initial $H$-algebra: due to $H \emptyset \neq \emptyset \neq \bar{H} \emptyset$, the two functors have the same categories of algebras.

Example 2.47. Let $H=C_{01}+C_{1}$ be the constant functor of value 2 except $\emptyset \mapsto 1$. The functor $\bar{H}$ in the proof above is the constant functor with value $1+1$, expressed, say as $\{a, b\}$. Here

$$
H \emptyset=\{b\} \quad \text { and } \quad H A=\{a, b\} \quad \text { for } A \neq \emptyset .
$$

The coalgebra

$$
\{a\} \smile\{a, b\}
$$

is obviously well-founded w.r.t. $\bar{H}$ but not w.r.t. $H$ since we have the pullback:

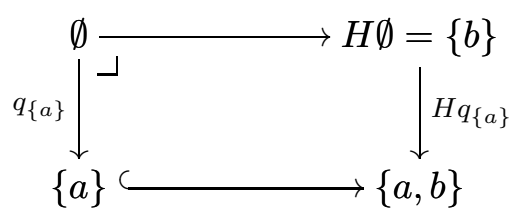

\subsection{The canonical graph and well-foundedness.}

Definition 2.48. Let $H$ be a set functor preserving (wide) intersections. For every coalgebra $a: A \longrightarrow H A$ define the canonical graph on $A$ : the neighbors of $x \in A$ are precisely those elements of $A$ which lie in the least subset $m: M \hookrightarrow A$ with $a(x) \in H m[H M]$.

Remark 2.49. (a) Gumm observed in [17] that if $H$ preserves intersections we obtain a "subnatural" transformation from it to the power-set functor $\mathscr{P}$ by defining functions

$$
\tau_{A}: H A \longrightarrow \mathscr{P} A, \quad \tau_{A}(x)=\text { the least subset } m: M \hookrightarrow A \text { with } x \in H m[H M] .
$$

The naturality squares do not commute in general, but for every monomorphism $m: A^{\prime}$ $\longrightarrow A$ we have a commutative square

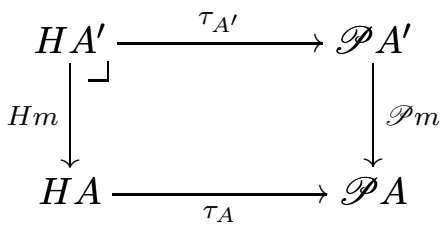

which even is a pullback. The canonical graph of a coalgebra $a: A \longrightarrow H A$ is simply the graph $\tau_{A} \cdot a: A \longrightarrow \mathscr{P} A$.

(b) Recall that a graph is well-founded iff it has no infinite directed paths. This also fully characterizes well-foundedness of $H$-coalgebras:

Proposition 2.50. If a set functor preserves intersections, then a coalgebra is well-founded iff its canonical graph is well-founded.

Remark. For functors $H$ preserving inverse images this fact is proved by Taylor [29, Remark 6.3.4]. Our proof is essentially the same. 
Proof. Let $a: A \longrightarrow H A$ be a well-founded coalgebra. Given a subgraph $\left(A^{\prime}, a^{\prime}\right)$ of the associated graph $(A, \tau \cdot a)$ forming a pullback

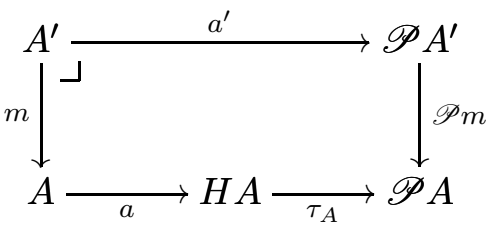

we are to prove that $m$ is invertible. Use the pullback of Remark 2.49.

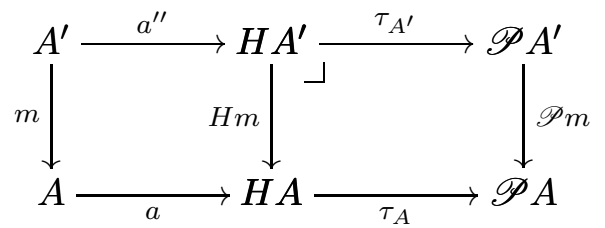

We get a unique $a^{\prime \prime}: A^{\prime} \longrightarrow H A^{\prime}$ with $a^{\prime}=\tau_{A^{\prime}} \cdot a^{\prime \prime}$, and $\left(A^{\prime}, a^{\prime \prime}\right)$ is a subcoalgebra of $(A, a)$. Moreover, in the diagram above the outside square and the right-hand one are both pullbacks, thus, the left-hand square is also a pullback. Consequently, $m$ is invertible since $(A, a)$ is well-founded.

Conversely, if the graph $\left(A, \tau_{A} \cdot a\right)$ is well-founded, we are prove that if the left-hand square of (2.20) is a pullback then $m$ is invertible. Indeed, in that case, by composition, the outside square is a pullback for the subcoalgebra $\left(A^{\prime}, \tau_{A^{\prime}} \cdot a^{\prime \prime}\right)$ of $\left(A, \tau_{A} \cdot a\right)$. Thus, since the last coalgebra is well-founded, $m$ is invertible.

\subsection{Initial algebras for functors on vector spaces.}

For every field $K$, the category $\mathrm{Vec}_{K}$ of vector spaces over $K$ also has the property that the equality (2.13) holds for all endofunctors. This follows from the next lemma whose proof is a variation of Trnková's proof of Proposition 2.43 (cf. [31]):

Lemma 2.51. In $\mathrm{Vec}_{K}$, finite intersections of monomorphisms are absolute, i.e., preserved by every functor with domain $\mathrm{Vec}_{K}$.

Corollary 2.52. For every endofunctor of $\mathrm{Vec}_{K}$ we have

$$
\text { initial algebra }=\text { final well-founded coalgebra. }
$$

Remark 2.53. The existence of an initial algebra is equivalent to the existence of a space $X \cong H X$, see Proposition 2.13.

\section{WELL-POINTED COALGEBRAS}

\subsection{Simple coalgebras.}

We arrive at the centerpiece of this paper, characterizations of the initial algebra, final coalgebra, and initial iterative algebra for endofunctors preserving intersections. Recall from Section 2 that subcoalgebras are represented by homomorphisms carried by monomorphisms in $\mathscr{A}$, and quotient coalgebras by homomorphisms carried by strong epimorphisms in $\mathscr{A}$. 
Here we prove that an endofunctor preserving monomorphisms has a final coalgebra iff it has only a set of simple coalgebras (up to isomorphism). For concrete categories and endofunctors preserving intersections we prove a stronger result: the final coalgebra consists of all well-pointed coalgebras which are those pointed coalgebras with no proper quotient and no proper subobject. And a much sharper result is obtained if the base category is an equational class of algebras. Numerous examples of this type of description of final coalgebras are presented in Section 4 .

Assumption 3.1. Throughout this section $\mathscr{A}$ denotes a cocomplete, wellpowered and cowellpowered category. And $H: \mathscr{A} \longrightarrow \mathscr{A}$ is an endofunctor.

Additionally, in a number of results below we assume that $H$ preserves (wide) intersections, i.e., multiple pullbacks of monomorphisms.

Examples 3.2. In the case where $\mathscr{A}=$ Set the assumption that $H$ preserves intersections is an extremely mild condition: examples include

(a) the power-set functor, all polynomial functors, the finite distribution functor,

(b) products, coproducts, quotients, and subfunctors of functors preserving intersections, and

(c) "almost" all finitary functors: if $H$ is finitary then $\bar{H}$ in Theorem 2.46 preserves intersections, see Lemma 2.45.

(d) An example of an important set functor not preserving intersections is the continuation $\operatorname{monad} X \mapsto R^{\left(R^{X}\right)}$ for a fixed set $R$.

\section{Remark 3.3.}

(a) We are working with factorizations of morphisms as strong epimorphisms followed by monomorphisms, see Remark 2.6. Recall from [4] that every cocomplete and cowellpowered category has such factorizations.

(b) We use Terminology 2.8 and recall from Remark 2.7 that quotients and subcoalgebras form a factorization system in Coalg $H$ whenever $H$ preserves monomorphisms. In this case, a coalgebra $(A, a)$ is simple (see Definition 2.1) iff every homomorphism from it is a subcoalgebra.

Notation 3.4. From now on we will write

$$
\nu H \quad \text { and } \quad \mu H
$$

for the final coalgebra and initial algebra for $H$, respectively, whenever they exist.

\section{Examples 3.5.}

(1) If $H$ has a final coalgebra $\nu H$, then $\nu H$ is simple. Indeed, the terminal object of every category is (clearly) simple.

(2) If a set functor $H$ has an initial algebra, then the corresponding coalgebra is simple (see Theorem 2.46). More generally, let $\mathscr{A}$ be an LFP category with a simple initial object. If $H$ preserves finite intersections and has an inital algebra $\mu H$, then $\mu H$ is (as a coalgebra) simple. Indeed, by Theorem 2.38, $\mu H$ is a final well-founded coalgebra. Since well-founded coalgebras are closed under quotients (see Lemma 2.28), it follows that $\mu H$ is simple (in Coalg $H$ ).

(3) A deterministic automaton considered as a coalgebra of

$$
H X=X^{I} \times\{0,1\} \quad(I=\text { the set of inputs })
$$


is simple iff it is observable. That is, every pair of distinct states accept distinct languages.

(4) A graph, considered as a coalgebra for $\mathscr{P}$, is simple iff it has pairwise non-bisimilar vertices.

Observation 3.6. Simple coalgebras form an ordered class (up to isomorphism), i.e., between two simple coalgebras there exists at most one homomorphism.

Indeed, given a parallel pair $h_{1}, h_{2}:(A, a) \longrightarrow(B, b)$, their coequalizer is a quotient of $(B, b)$, hence it is invertible and we conclude $h_{1}=h_{2}$.

Proposition 3.7 (Gumm [16]). Every coalgebra has a unique simple quotient represented by the wide pushout

$$
e_{(A, a)}:(A, a) \longrightarrow(\bar{A}, \bar{a})
$$

of all quotients. If $H$ preserves monomorphisms, this is the reflection of $(A, a)$ in the full subcategory of all simple coalgebras.

Gumm worked with $\mathscr{A}=$ Set, but his argument extends without problems: for every coalgebra homomorphism $f:(A, a) \longrightarrow(B, b)$ there exists a unique coalgebra homomorphism $\bar{f}:(\bar{A}, \bar{a}) \longrightarrow(\bar{B}, \bar{b})$ with $\bar{f} \cdot e_{(A, a)}=e_{(B, b)} \cdot f$ by the universal property of wide pushouts.

Corollary 3.8. Every subcoalgebra of a simple coalgebra is simple.

Indeed, every full (strong epi)-reflective subcategory is closed under subobjects.

Theorem 3.9. For every endofunctor $H$ the existence of $\nu H$ implies that $H$ has only a set of simple coalgebras (up to isomorphism). If $H$ preserves monomorphisms, the converse implication also holds.

Remark. Moreover, if $\left(A_{i}, a_{i}\right), i \in I$, is a set of representatives of all simple coalgebras, then $\nu H$ is the simple quotient of their coproduct:

$$
\nu H=(\bar{A}, \bar{a}) \quad \text { where } \quad(A, a)=\coprod_{i \in I}\left(A_{i}, a_{i}\right) .
$$

The theorem is a consequence of Freyd's Adjoint Functor Theorem. We include a (short) proof for the convenience of the reader.

Proof. (1) Let $H$ have a set $\left(A_{i}, a_{i}\right), i \in I$, of representative simple coalgebras. Proposition 3.7 implies that this set is weakly final: for every coalgebra $(B, b)$ choose $i \in I$ with $(\bar{B}, \bar{b}) \simeq\left(A_{i}, a_{i}\right)$ and obtain a homomorphism $(B, b) \longrightarrow\left(A_{i}, a_{i}\right)$. Consequently, the coproduct $(A, a)$ above is a weakly final object, hence, so is its quotient $(\bar{A}, \bar{a})$. For every parallel pair of morphisms with codomain $(\bar{A}, \bar{a})$ their coequalizer is invertible (since the codomain is simple, see Remark 3.3$)$. Hence, $(\bar{A}, \bar{a})$ is final.

(2) Let $\nu H$ exist. Then for every simple coalgebra the unique homomorphism into $\nu H$ is monic. Therefore, since $\mathscr{A}$ is wellpowered by assumption, $H$ has only a set of simple coalgebras up to isomorphism. 
Example 3.10. If $H$ does not preserve monomorphisms, then it can have both a final coalgebra and a proper class of simple coalgebras which are pairwise non-isomorphic.

On the category Gra of graphs and graph morphisms define an endofunctor, based on the power-set functor $\mathscr{P}$, as follows:

$$
H X= \begin{cases}\mathscr{P} X \text { (no edges) } & \text { if } X \text { has no edges } \\ 1 & \text { else }\end{cases}
$$

For morphisms between graphs without edges put $H f=\mathscr{P} f$. Then $1=H 1$ is the final coalgebra.

Now $\mathscr{P}$ as an endofunctor of Set has, since no final coalgebra exists, a proper class of simple, pairwise non-isomorphic coalgebras $a_{i}: A_{i} \longrightarrow \mathscr{P} A_{i}(i \in I)$. Consider $A_{i}$ as a graph without edges, then $\left(A_{i}, a_{i}\right)$ is a coalgebra for $H$. And this coalgebra is simple because if a coalgebra homomorphism $e:\left(A_{i}, a_{i}\right) \longrightarrow(B, b)$ is carried by a strong epimorphism $e: A_{i} \longrightarrow B$ of Gra, then the fact that $A_{i}$ has no edge implies that neither has $B$. Then $e$ is a homomorphism in Coalg $\mathscr{P}$ which implies that it is invertible (in Set, hence, in Gra). Thus, we obtain a proper class of simple $H$-coalgebras $\left(A_{i}, a_{i}\right)$.

\subsection{Well-pointed coalgebras.}

Remark 3.11. In the rest of Section 3 we assume that the base category $\mathscr{A}$ is concrete, i.e., a faithful functor

$$
U: \mathscr{A} \longrightarrow \text { Set }
$$

is given. We require that $U$

(a) preserves intersections,

(b) is fibre-small, i.e., for every set $X$ there exists up to isomorphism only a set of objects $A \in \mathscr{A}$ with $U A=X$, and

(c) is uniquely transportable, i.e., for every object $A$ and every bijection $b: U A \longrightarrow X$ in Set there exists a unique object $\bar{A}$ with $U \bar{A}=X$ and $A \simeq \bar{A}$ where the isomorphism is carried by $b$.

Condition (c) is harmless: every concrete category is equivalent to a uniquely transportable one, see [4, Proposition 5.36]. Also (a) and (b) are conditions fulfilled by all "everyday" concrete categories: usually $U$ is the hom-functor of an object $G$ which is a generator, and then (a) and (b) hold. More generally:

\section{Example 3.12.}

(1) Let $G_{i}(i \in I)$ be a generating set of $\mathscr{A}$, i.e., for every parallel pair $f_{1}, f_{2}: A \longrightarrow B$ of distinct morphisms there exists $i \in I$ and $h: G_{i} \longrightarrow A$ with $f_{1} \cdot h \neq f_{2} \cdot h$. Then the functor

$$
U=\coprod_{i \in I} \mathscr{A}\left(G_{i},-\right): \mathscr{A} \longrightarrow \text { Set }
$$

is faithful, fibre-small, and preserves intersections. Indeed, faithfulness is equivalent to $\left\{G_{i}: i \in I\right\}$ forming a generating set. Each $\mathscr{A}\left(G_{i},-\right)$ preserves limits, and connected limits commute with coprodutcs in Set, thus, $U$ preserves connected limits. Fibresmallness follows from $\mathscr{A}$ being cocomplete and cowellpowered: for every object $A$ the canonical morphism

$$
e: \coprod_{i \in I} \mathscr{A}\left(G_{i}, A\right) \bullet G_{i} \longrightarrow A
$$


where $-\bullet G_{i}$ denotes copowers of $G_{i}$ (and the $f$-component of $e$ is $f$ for every $f \in$ $\left.\mathscr{A}\left(G_{i}, A\right)\right)$ is an epimorphism. This is also equivalent to $\left\{G_{i}: i \in I\right\}$ forming a generating set. For every set $X$ all objects $A$ with $U A=X$ are thus quotients of $\coprod_{i \in I} X_{i} \bullet G_{i}$ where $X_{i} \subseteq X$. Since $\mathscr{A}$ is cowellpowered, all these quotients form a set of objects up to isomorphism.

(2) Every LFP category $\mathscr{A}$ is concrete as described in the previous point when one chooses as generating set any set of representatives of all finitely presentable objects up to isomorphism.

Definition 3.13. By a pointed coalgebra is meant a triple $(A, a, x)$ consisting of a coalgebra $a: A \longrightarrow H A$ and an element $x$ of $U A$. The category

\section{$\operatorname{Coalg}_{p} H$}

of pointed coalgebras has as morphisms from $(A, a, x)$ to $(B, b, y)$ those coalgebra homomorphisms $f:(A, a) \longrightarrow(B, b)$ which preserve the point:

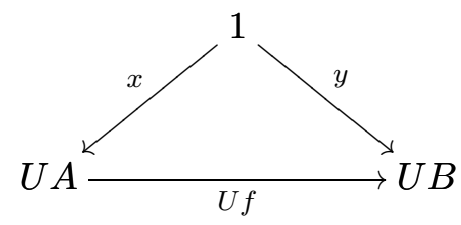

Remark 3.14. As for Coalg $H$, the quotients of a pointed coalgebra $(A, a, x)$ are precisely the morphisms with this domain carried by strong epimorphisms of $\mathscr{A}$. And subcoalgebras are precisely the morphisms with codomain $(A, a, x)$ carried by monomorphisms of $\mathscr{A}$. Moreover, Remark 2.7 immediately extends to Coalg $_{p} H$.

Definition 3.15. A well-pointed coalgebra is a pointed coalgebra with no proper quotient and no proper subobject.

\section{Remark.}

(a) To say that a pointed coalgebra $(A, a, x)$ has no proper subobject means precisely that $x$ generates the coalgebra $(A, a)$ : whenever a subcoalgebra $m:(B, b) \longrightarrow(A, a)$ contains $x$ (in the image of $U m$ ) then $m$ is invertible. We call such coalgebras reachable. Thus:

$$
\text { well-pointed }=\text { simple }+ \text { reachable. }
$$

(b) It is easy to see that if $f:(A, a, x) \longrightarrow(B, b, y)$ is a morphism of pointed coalgebras, and if $(A, a)$ is simple and $(B, b, y)$ is reachable, then $f$ is an isomorphism.

(c) In the case where $\mathscr{A}=$ Set and $H=\mathscr{P}$, reachability of a pointed graph means that every vertex can be reached from the chosen one. Suppose $H$ is an arbitrary set functor preserving intersections. Then reachability of coalgebras can be translated to reachability of its canonical graph, see Definition 2.48,

Lemma 3.16. Let $H$ be a set functor preserving intersections. Then a pointed coalgebra $(A, a, x)$ is reachable iff its pointed canonical graph is, i.e., every vertex can be reached from $x$ by a directed path. 
Proof. Recall $\tau_{A}: H A \longrightarrow \mathscr{P} A$ from Remark 2.49, Take a subcoalgebra $\left(A^{\prime}, a^{\prime}\right)$ containing $x$ :

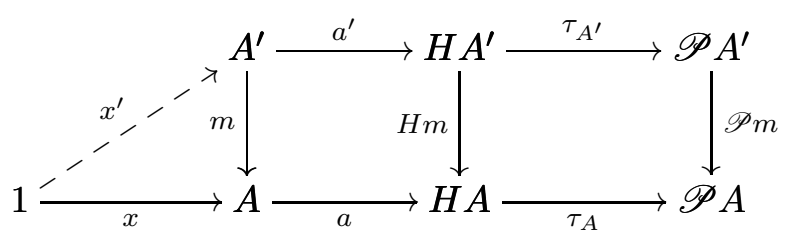

Then $A^{\prime}$ is a subcoalgebra of the canonical graph $\left(A, \tau_{A} \cdot a\right)$ (as a pointed coalgebra of $\mathscr{P}$ ). And vice versa: if $m: A^{\prime} \longrightarrow A$ is a subobject of the pointed canonical graph then, since the square in Remark 2.49 is a pullback, we have a unique structure $a^{\prime}: A^{\prime} \longrightarrow H A^{\prime}$ of a subobject of $(A, a, x)$. Therefore, $(A, a, x)$ is reachable w.r.t. $H$ iff $\left(A, \tau_{A} \cdot a, x\right)$ is reachable w.r.t. $\mathscr{P}$.

\section{Examples 3.17.}

(a) A deterministic automaton with a given initial state is a pointed coalgebra for $H X=$ $X^{I} \times\{0,1\}$. Reachability means that every state can be reached (in finitely many steps) from the initial state. The usual terminology is that reachability and observability (see Example 3.5 (3)) together are called minimality. Thus, well-pointed coalgebras are precisely the minimal automata.

(b) For the power-set functor the pointed coalgebras are the pointed graphs. Well-pointed means reachable and simple (Example 3.5 (4)). See Subsection 4.6 for more details.

Notation 3.18. If $H$ preserves intersections, then there is a canonical process of turning an arbitrary pointed coalgebra $(A, a, x)$ into a well-pointed one: form the simple quotient (see Proposition 3.7 ) pointed by $U e_{(A, a)} \cdot x: 1 \longrightarrow U \bar{A}$, then form the least subcoalgebra containing that point:
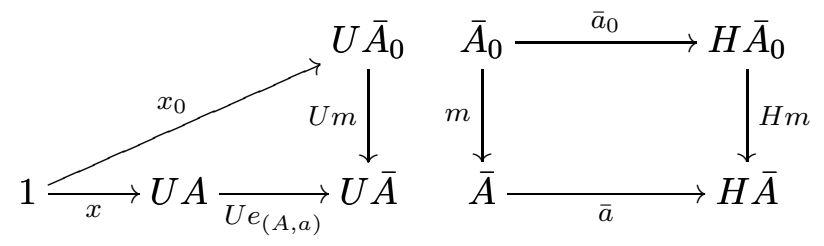

Then $\left(\bar{A}_{0}, \bar{a}_{0}, x_{0}\right)$ is well-pointed by Corollary [3.8. We denote the well-pointed coalgebra $\left(\bar{A}_{0}, \bar{a}_{0}, x_{0}\right)$ (unique up to isomorphism ) by

$$
\mathrm{wp}(A, a, x)
$$

and call it the well-pointed modification of $(A, a, x)$.

Example 3.19. For deterministic automata our process $A \longmapsto \bar{A}_{0}$ above means that we first merge the states that are observably equivalent and then discard the states that are not reachable. A more efficient way is first discarding the unreachable states and then merging observably equivalent pairs. Both ways are possible if our functor preserves inverse images:

Remark 3.20. Let $H$ and $U$ preserve inverse images. Then a quotient of a reachable pointed coalgebra is reachable. Indeed, given such a quotient $e$ and its subcoalgebra $m$ containing the given point $x$, form the inverse image $m^{\prime}$ of $m$ along $e$, and apply $U$ to this 
pullback:

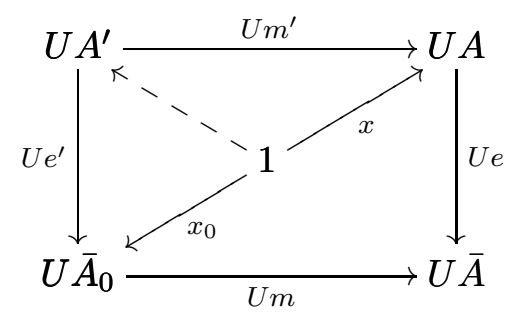

Since $H$ preserves inverse images, $m^{\prime}: A^{\prime} \longrightarrow A$ is a subcoalgebra of $A$, and, since $U$ preserves inverse images too, the universal property of pullbacks implies that $A^{\prime}$ contains the given point $x$. Consequently, $m^{\prime}$ is invertible, thus, $m \cdot e^{\prime}$ is strongly epic, therefore $m$ is invertible.

Thus, we have an alternative procedure of forming well-pointed coalgebras from pointed ones, $(A, a, x)$ : first form the least pointed subcoalgebra $\left(A_{0}, a_{0}, x\right)$. Then form the simple quotient of $\left(A_{0}, a_{0}\right)$.

Notation 3.21. The collection of all well-pointed coalgebras up to isomorphism is denoted by $T$. For every coalgebra $a: A \longrightarrow H A$ we have a function

$$
a^{+}: U A \longrightarrow T \quad \text { defined by } \quad a^{+}(x)=\mathrm{wp}(A, a, x) .
$$

(Notice that the well-pointed modification $\operatorname{wp}(A, a, x)$ is unique up to isomorphism. Thus we have precisely one choice in $T$.)

Lemma 3.22. Let $H$ preserve monomorphisms. For every coalgebra homomorphism $h$ : $(A, a) \longrightarrow(B, b)$ the triangle

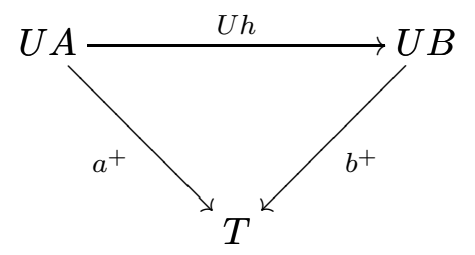

commutes.

Proof. (a) Assume that both coalgebras above are simple. In particular, $h$ is a monomorphism by simplicity of $(A, a)$. For every element $x \in U A$ we know that $a^{+}(x)$ is the subcoalgebra $m:\left(A_{0}, a_{0}\right) \longrightarrow(A, a)$ generated by $x$. Therefore $h \cdot m:\left(A_{0}, a_{0}\right) \longrightarrow(B, b)$ is a subcoalgebra of $(B, b, U h(x))$, and since $\left(A_{0}, a_{0}, x_{0}\right)$, with $U m\left(x_{0}\right)=x$, is well-pointed, we conclude that it is isomorphic to $b^{+}(U h(x))$. Now $T$ contains just one representative of every well-pointed coalgebra up to isomorphism, consequently, $b^{+}(U h(x))=a^{+}(x)$.

(b) If the two coalgebras are arbitrary, form the reflection $\bar{h}$ of $h$ (see Proposition 3.7):

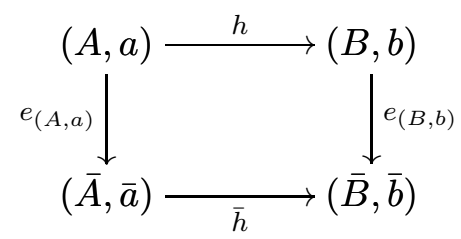

Then for every element $x \in U A$ we have that $a^{+}(x)$ is the subcoalgebra of $(\bar{A}, \bar{a})$ generated by $\bar{x}=U e_{(A, a)}(x)$, thus $a^{+}(x)=\bar{a}^{+}(\bar{x})$; analogously for $b^{+}(U h(x))$. By applying (a) to $\bar{h}$ in lieu of $h$ we conclude $a^{+}(x)=\bar{a}^{+}(\bar{x})=\bar{b}^{+}(U \bar{h}(\bar{x}))=b^{+}(U h(x))$. 
Lemma 3.23. If $(A, a)$ is a simple coalgebra, then $a^{+}: U A \longrightarrow T$ is injective.

Proof. Suppose that $\operatorname{wp}\left(A, a, x^{1}\right)=\operatorname{wp}\left(A, a, x^{2}\right)$. Let $m_{i}:\left(A_{i}, a_{i}, x_{0}^{i}\right) \longrightarrow\left(A, a, x^{i}\right)$ denote the smallest subcoalgebra containing $x^{i}(i=1,2)$ which is isomorphic to $\operatorname{wp}\left(A, a, x^{i}\right)$. Let

$$
u:\left(A_{1}, a_{1}, x_{0}^{1}\right) \longrightarrow\left(A_{2}, a_{2}, x_{0}^{2}\right)
$$

be an isomorphism. Then since $(A, a)$ is simple, we have $m_{1}=m_{2} \cdot u$ due to Observation 3.6. From $U u\left(x_{0}^{1}\right)=x_{0}^{2}$ we get

$$
x^{2}=U m_{2}\left(x_{0}^{2}\right)=U\left(m_{2} u\right)\left(x_{0}^{1}\right)=U m_{1}\left(x_{0}^{1}\right)=x^{1} .
$$

\subsection{Final coalgebras.}

We remind the reader that in this section, we assume that the endofunctor $H$ preserves intersections.

Theorem 3.24. $H$ has a final coalgebra iff it has only a set of well-pointed coalgebras up to isomorphism. Moreover, a set $T$ of representatives of well-pointed coalgebras carries the final coalgebra:

$$
U(\nu H)=T \text {. }
$$

Remark 3.25. The final coalgebra for $H$ is, as we will also prove, characterized up to isomorphism as a coalgebra $\bar{\tau}: \bar{T} \longrightarrow H \bar{T}$ with two properties: $U \bar{T}=T$, and for every coalgebra $(A, a)$ the function $a^{+}: U A \longrightarrow T$ carries a coalgebra homomorphism from $(A, a)$ to $(\bar{T}, \bar{\tau})$.

Proof. The necessity follows from Theorem 3.9. For the sufficiency, fix a set $T$ of representative well-pointed coalgebras. We also use Theorem 3.9 to show that $H$ has a final coalgebra. Indeed, if $(A, a)$ is a simple coalgebra, then by Lemma 3.23, $U A$ has cardinality at most card $T$. Since $\mathscr{A}$ is small-fibred and uniquely transportable, it has up to isomorphism of $\mathscr{A}$ only a set of objects whose underlying sets have cardinality at most card $T$. Consequently, $H$ has up to isomorphism of Coalg $H$ only a set of simple coalgebras: given an object $A$ with $\mathscr{A}(A, H A)$ of cardinality $\alpha$, there are at most $\alpha$ pairwise non-isomorphic coalgebras $b: B \longrightarrow H B$ with $A \cong B$ in $\mathscr{A}$.

Given the coalgebra structure

$$
\tau: \nu H \longrightarrow H(\nu H)
$$

of the final coalgebra, we now prove that the map $\tau^{+}: U(\nu H) \longrightarrow T$ is a bijection. Indeed, $\tau^{+}$is monic due to the simplicity of $\nu H$ (see Example3.5(1)) and Lemma3.23. To check the surjectivity, let $a^{+}(x) \in T$, where $(A, a)$ is a coalgebra and $x \in U A$. Then by Lemma 3.22, $a^{+}(x)=\tau^{+}(U h(x))$, where $h: A \longrightarrow \nu H$ is the coalgebra homomorphism. This shows that the image of $\tau^{+}$contains $a^{+}(x)$.

Since $\mathscr{A}$ is uniquely transportable, there exists a unique object $\bar{T}$ of $\mathscr{A}$ and a unique isomorphism $i: \nu H \longrightarrow \bar{T}$ with $\tau^{+}=U i$. Define a coalgebra $\bar{\tau}: \bar{T} \longrightarrow H \bar{T}$ so that $i$ is a coalgebra isomorphism: $\bar{\tau}=H i \cdot \tau \cdot i^{-1}$. The coalgebra $(\bar{T}, \bar{\tau})$ is final because for every coalgebra $(A, a)$ we have a unique coalgebra homomorphism $a^{*}:(A, a) \longrightarrow(\nu H, \tau)$, hence 
a unique coalgebra homomorphism $i \cdot a^{*}:(A, a) \longrightarrow(\bar{T}, \bar{\tau})$ :

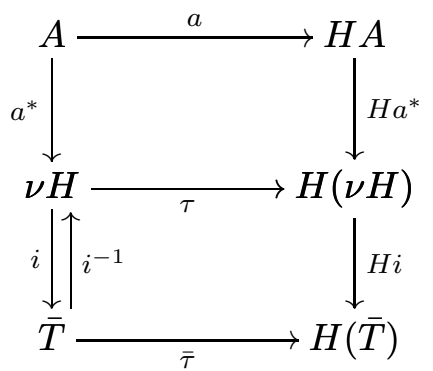

We conclude with the verification of Remark 3.25. First we show that $\bar{\tau}^{+}=$id. To see this apply Lemma 3.22 to $i$ in order to get $\tau^{+}=\bar{\tau}^{+} \cdot U i$. But since $\tau^{+}=U i$, we get $U i=\bar{\tau}^{+} \cdot U i$, hence $\bar{\tau}^{+}=$id because $U i$ is an isomorphism.

To see that $a^{+}=U\left(i \cdot a^{*}\right)$, we use Lemma 3.22 again:

$$
a^{+}=\bar{\tau}^{+} \cdot U\left(i \cdot a^{*}\right)=\mathrm{id} \cdot U\left(i \cdot a^{*}\right)=U\left(i \cdot a^{*}\right) .
$$

For the uniqueness, suppose that $\left(T^{\prime}, \tau^{\prime}: T^{\prime} \longrightarrow H T^{\prime}\right)$ also fulfils $U T^{\prime}=T$ and for all coalgebras $(A, a)$, the map $a^{+}: U A \longrightarrow T$ is $U(b)$ for some coalgebra homomorphism $b:(A, a) \longrightarrow\left(T^{\prime}, \tau^{\prime}\right)$. We apply this with $(A, a)=(\bar{T}, \bar{\tau})$, and so id $=\bar{\tau}^{+}: T \longrightarrow T$ is $U f$ for some coalgebra morphism $f:(\bar{T}, \bar{\tau}) \longrightarrow\left(T^{\prime}, \tau^{\prime}\right)$. However, by unique transportability, there is some isomorphism $g: \bar{T} \longrightarrow T^{\prime}$ such that $U g=$ id. And by faithfulness, $f=g$. Thus the coalgebras $(\bar{T}, \bar{\tau})$ and $\left(T^{\prime}, \tau^{\prime}\right)$ are isomorphic.

Example 3.26. Let $H$ be a set functor preserving intersections. If $T$ is a set of representatives of all well-pointed coalgebras, then $T$ is a final coalgebra. Its coalgebra structure assigns to every member $(A, a, x)$ of $T$ the following member of $H T$ :

$$
1 \stackrel{x}{\longrightarrow} A \stackrel{a}{\longrightarrow} H A \stackrel{H a^{+}}{\longrightarrow} H T .
$$

See Section 4 for numerous concrete examples.

Example 3.27. If $H$ does not preserve intersections the theorem can fail: the functor in Example 3.10 has a proper class of well-pointed coalgebras.

Example 3.28. For the set functor

$$
H X=X^{I} \times\{0,1\}
$$

presenting deterministic automata the well-pointed coalgebras are precisely the minimal (i.e., reachable and observable $=$ simple) automata. Since every language $L \subseteq I^{*}$ is accepted by a minimal automaton, unique up to isomorphism, we get the more usual description of the final coalgebra

$$
\begin{aligned}
\nu X \cdot X^{I} \times\{0,1\} & =\text { all minimal automata } \\
& \cong \mathscr{P} I^{*} \text { (all languages) }
\end{aligned}
$$

Remark 3.29. Actually every set functor $H$ has a final coalgebra, but this can be a proper class. More precisely, $H$ has an extension $H^{*}$ to the category of classes and functions unique up to a natural isomorphism, and $\nu H^{*}$ exists, see [7].

Corollary 3.30. For every set functor $H$ preserving intersections a class of representative well-pointed $H$-coalgebras with the coalgebra structure given by the formula (3.1) is a final coalgebra for $H^{*}$. 
The proof is completely analogous to that of Theorem 3.24 .

Example 3.31. The final coalgebra of the power set functor is the class of all well-pointed graphs (up to isomorphism).

Construction 3.32. Now let $\mathscr{A}$ be a variety of algebras determined by a set $E$ of equations, for a fixed signature $\Sigma$. Given a set $T$ representing all well-pointed coalgebras up to isomorphism, we turn it into a final coalgebra of $H$ as follows.

(a) $T$ as a $\Sigma$-algebra. For every $n$-ary symbol $\sigma \in \Sigma$ define $\sigma^{T}: T^{n} \longrightarrow T$ as follows: Given an $n$-tuple of elements $\left(A_{i}, a_{i}, x_{i}\right)$ of $T$ form a coproduct

$$
A=\coprod_{i<n} A_{i} \quad \text { in } \mathscr{A}
$$

and obtain a coproduct $(A, a)=\coprod_{i<n}\left(A_{i}, a_{i}\right)$ in Coalg $H$ together with elements $\hat{x}_{i} \in A$ corresponding to $x_{i} \in A_{i}$. For the element

$$
y=\sigma^{A}\left(\hat{x}_{i}\right)_{i<n} \text { of } A
$$

we define the result of $\sigma^{T}$ as the well-pointed modification of $(A, a, y)$ :

$$
\sigma^{T}\left(A_{i}, a_{i}, x_{i}\right)_{i<n}=\mathrm{wp}(A, a, y) .
$$

(b) $T$ will be proved to satisfy all equations in $E$, i.e., $T$ is an object of $\mathscr{A}$. And all $a^{+}$in Notation 3.21 are $\Sigma$-homomorphisms. (See Lemma 3.33.)

(c) $T$ as a coalgebra. We have a function

$$
\tau: T \longrightarrow H T
$$

defined precisely as in Example 3.26 .

$$
\tau(A, a, x)=H a^{+}(a(x))
$$

We prove that $\tau$ is a $\Sigma$-homomorphism. (See Proposition 3.34.)

(d) We derive that $(T, \tau)$ is a final coalgebra for $H$.

Lemma 3.33. The $\Sigma$-algebra $T$ lies in $\mathscr{A}$ and for every coalgebra $(A, a)$ we have a $\Sigma$ homomorphism $a^{+}: A \longrightarrow T$.

Proof. Recall the final coalgebra $(\bar{T}, \bar{\tau})$ from the proof of Theorem 3.24 whose underlying set is $T$. All we need to prove is that the operations $\sigma^{\bar{T}}$ of the $\Sigma$-algebra $\bar{T}$ are given by the formula (3.2) above. Indeed, given a well-pointed coalgebra $\left(A_{i}, a_{i}, x_{i}\right)$ we have $a_{i}^{+}\left(x_{i}\right)=\left(A_{i}, a_{i}, x_{i}\right)$. Let us apply Lemma 3.22 to the coproduct injection $v_{i}:\left(A_{i}, a_{i}\right)$ $\longrightarrow(A, a)$ : since $a_{i}^{+}=a^{+} \cdot v_{i}$ and $\hat{x}_{i}=v_{i}\left(x_{i}\right)$, we conclude

$$
\left(A_{i}, a_{i}, x_{i}\right)=a_{i}^{+}\left(x_{i}\right)=a^{+}\left(\hat{x}_{i}\right)
$$

Since $a^{+}: A \longrightarrow \bar{T}$ is (by Remark 3.25) a $\Sigma$-homomorphism, we obtain

$$
\sigma^{\bar{T}}\left(\left(A_{i}, a_{i}, x_{i}\right)_{i<n}\right)=\sigma^{\bar{T}}\left(\left(a^{+}\left(\hat{x}_{i}\right)\right)_{i<n}\right)=a^{+}\left(\sigma^{A}\left(\left(\hat{x}_{i}\right)_{i<n}\right)\right)=a^{+}(y)=\mathrm{wp}(A, a, y)
$$

as required. 
Proposition 3.34. The function $\tau(A, a, x)=H a^{+}(a(x))$ is a $\Sigma$-homomorphism from $\mathrm{T}$ to $H T$, and the coalgebra $(T, \tau)$ is final.

Proof. For the final coalgebra $\bar{\tau}: \bar{T} \longrightarrow H \bar{T}$ of the proof of Theorem 3.24 we already know that $T=\bar{T}$. It remains to prove that $\bar{\tau}=\tau$. For every element $(A, a, x)$ of $T$ we have $a^{+}(x)=(A, a, x)$ and, since $a^{+}:(A, a) \longrightarrow(T, \bar{\tau})$ is by Remark 3.25 a coalgebra homomorphism,

$$
\bar{\tau}(A, a, x)=\bar{\tau}\left(a^{+}(x)\right)=H a^{+}(a(x))=\tau(A, a, x) .
$$

\section{Remark 3.35.}

(a) Generalizing deterministic automata, see Example 3.28, every pointed coalgebra $(A, a, x)$ can be viewed as a realization of the corresponding element $t=a^{+}(x)$ of the terminal coalgebra of $H$. The well-pointed coalgebras are the minimal realizations of $t$. Then every element of $\nu H$ has a minimal realization, unique up-to isomorphism.

(b) If the algebra $A$ above is finite, then minimality is equivalent to state-minimality:

(b1) Every realization of $t$ has cardinality at least that of $A$, and

(b2) if it has the same cardinality as $A$, it is isomorphic to $(A, a, x)$.

This follows from the fact that every well-pointed coalgebra $(A, a, x)$ is, as we have seen above, isomorphic to the subcoalgebra of $\nu H$ generated by $a^{+}(x)$.

(c) For non-deterministic automata we obtain minimization w.r.t. bisimilarity (i.e. w.r.t. to the branching behavior) but this is not minimization in the classical sense. The reason is that the terminal coalgebra of the corresponding functor $\left(P_{f} X\right)^{I} \times\{0,1\}$ is not the set of all languages over $I$.

Example 3.36. In the variety Bool of boolean algebras consider the functor $H X=X^{I} \times \mathcal{Z}$ where $\mathcal{Z}$ is the 2 -element boolean algebra. Its coalgebras are deterministic automata with a boolean algebra structure on the states such that (1) final states form an ultrafilter, and (2) transitions preserve the boolean operations. The terminal coalgebra is the boolean algebra of all well-pointed coalgebras, and this is isomorphic to the boolean algebra of all languages over $I$.

For every regular language $L$ the unique minimal realization $A$ (i.e. the corresponding well-pointed H-coalgebra) is finite, but possibly larger than the minimal automaton in Set. However, by restricting ourselves to the atoms of the boolean algebra $A$, one obtains a nondeterministic automaton which Brzozowski and Tamm [11] call the átomaton for $L$ and which in some cases is the state-minimal nondeterministic realization of $L$ (in the classical sense in Set).

\subsection{Initial algebras.}

Assumption 3.37. In the rest of this section $\mathscr{A}$ denotes an LFP category with a simple initial object, and $H$ an endofunctor preserving intersections.

Just as the final coalgebra $\nu H$ for a set functor $H$ consists of all well-pointed coalgebras (up to isomorphism), we now prove that the initial algebra $\mu H$ consists of all well-founded, well-pointed coalgebras. In more detail: the well-founded coalgebras in $\nu H$ form a subcoalgebra, and we prove that this is a final well-founded coalgebra which by Theorem 2.38 is $\mu H$. In Section 4 we then present numerous examples of initial algebras described in this manner. 
Notation 3.38. Recall the concept of well-founded coalgebra from Section 2, The collection of all well-founded, well-pointed coalgebras (up to isomorphism) is denoted by $I$. For every well-founded coalgebra $a: A \longrightarrow H A$ we have a function

$$
a^{+}: \bigcup A \longrightarrow I
$$

assigning to every element $x: 1 \longrightarrow \bigcup A$ the well-founded, well-pointed coalgebra of Notation 3.18

$$
a^{+}(x)=\left(\bar{A}_{0}, \bar{a}_{0}, x_{0}\right) .
$$

Indeed, $\left(\bar{A}_{0}, \bar{a}_{0}\right)$ is well-founded due to Corollary 2.28 and Lemma 2.29 .

Remark 3.39. Observe that for a pointed coalgebra to be well-founded and well-pointed two types of proper subcoalgebras are prohibited: the cartesian ones, and those containing the chosen point.

Theorem 3.40. $H$ has an initial algebra iff it has only a set of well-founded, well-pointed coalgebras up to isomorphism. Moreover a set I of representatives of well-founded, wellpointed coalgebras carries the initial algebra: $\bigcup(\mu H)=I$.

Proof. (1) If $H$ has an initial algebra $\mu H$, then by Theorem 2.38 this is a final well-founded coalgebra. Every well-founded, well-pointed coalgebra, being in particular simple, is a subcoalgebra of $\mu H$, since the unique homomorphism into $\mu H$ is carried by a monomorphism. Consequently, $I$ is a set.

(2) Let $H$ have a set $I$ of representatives of well-founded, well-pointed coalgebras. $I$ carries a canonical coalgebra structure

$$
\bar{\psi}: I \longrightarrow H I \text {. }
$$

As in Theorem 3.24, this structure assigns to every member $(A, a, x)$ of $I$ the following element of $\bigcup H I$ :

$$
1 \stackrel{x}{\longrightarrow} \bigcup A \stackrel{\cup a}{\longrightarrow} \bigcup H A \stackrel{\cup H a^{+}}{\longrightarrow} \bigcup H I
$$

We prove below that this is a final well-founded coalgebra. Thus, by Theorem 2.38, $I$ is an initial algebra w.r.t. the inverse of $\bar{\psi}$.

The proof that for every well-founded coalgebra $(A, a)$ the map $a^{+}: \bigcup A \longrightarrow I$ carries a unique coalgebra homomorphism into $\bar{\psi}: I \longrightarrow H(I)$ is completely analogous to the proof of Theorem 3.24. Just recall that subcoalgebras and quotients of a well-founded coalgebra are all well-founded (by Corollary 2.28 and Lemma 2.29).

It remains to prove that $(I, \bar{\psi})$ is a well-founded coalgebra. To this end notice that for every well-pointed, well-founded coalgebra $(A, a, x)$ in $I$ we have that

$$
a^{+}(x)=(A, a, x) \text {. }
$$

Now take the coproduct (in Coalg $H$ ) of all $(A, a)$ for which there is an $x \in A$ such that $(A, a, x)$ lies in $I$. This coproduct is a well-founded coalgebra by Corollary 2.28, and, as we have just seen, the unique induced homomorphism from this coproduct into $(I, \bar{\psi})$ is epimorphic, whence $I$ is a quotient coalgebra of the coproduct. Thus, another application of Corollary 2.28 shows that $(I, \bar{\psi})$ is a well-founded coalgebra as desired. 
Example 3.41. The initial algebra for $H X=X^{I} \times\{0,1\}$, and more generally, for any set functor $H$ with $H \emptyset=\emptyset$, is empty. No non-empty coalgebra is well-founded (due to the cartesian subcoalgebra $\emptyset$ ) and thus no pointed coalgebra is well-founded.

Remark 3.42. Analogously to Corollary 3.30, every set functor $H$ has a, possibly large, initial algebra. That is, the extension $H^{*}$ of $H$ to classes always has an initial algebra:

$$
\mu H^{*}=\text { all well-founded, well-pointed algebras }
$$

(up to isomorphism). This is a subcoalgebra of $\nu H$ of Remark 3.29. And as an algebra for $H^{*}$ it is initial:

Corollary 3.43. For every intersection preserving set functor $H$ the large coalgebra $\mu H^{*}$ is the final well-founded coalgebra for $H^{*}$. Thus, the large initial algebra is $\mu H^{*}$ w.r.t. the inverse of $\bar{\psi}$.

The first statement follows from the Small Subcoalgebra Lemma of 2 and the fact that subcoalgebras of well-founded coalgebras are well-founded (Corollary 3.8). The second statement is proved precisely as Theorem 2.38 .

Example 3.44. The initial algebra for $\mathscr{P}$ consists of all well-founded, well-pointed graphs.

Remark 3.45. The above theorem generalizes to endofunctors of finitary varieties having a simple initial object (and thus satisfying Assumptions 2.3). Let $\mathrm{H}$ be an endofunctor preserving intersections. Given a set $I$ representing well-founded, well-pointed coalgebras, we turn $I$ into a coalgebra of $H$ as in Construction 3.32, using the fact that (by Proposition 2.27) well-founded coalgebras are closed under coproducts. The rest of the proof is, due to Theorem 2.38, completely analogous to Proposition 3.34.

\subsection{Initial iterative algebras.}

In this subsection, we study another subcoalgebra of the final coalgebra for a set functor: all finite well-pointed coalgebras. We prove that this is the initial iterative algebra (also known as the rational fixed point). Before doing so we recall what completely iterative and iterative algebras are. Once again, there is no problem in generalizing the results below to locally finitely presentable base categories with a simple initial object and which are concrete via a given $U: \mathscr{A} \longrightarrow$ Set.

Remark 3.46. We know, from Theorem 2.38 and 3.40, that $\mu H$ has a double role: an initial algebra and a final well-founded coalgebra. Also $\nu H$ has a double role. Recall from [19] that an algebra $a: H A \longrightarrow A$ is completely iterative if for every (equation) morphism $e: X$ $\longrightarrow H X+A$ there exists a unique solution, i.e., a unique morphism $e^{\dagger}: X \longrightarrow A$ such that the square below commutes:

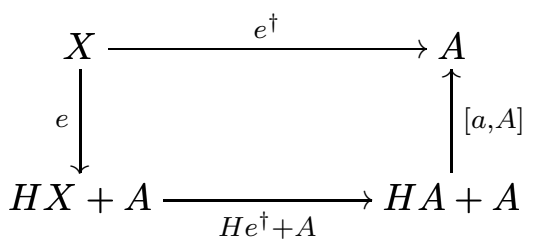

Theorem 3.47 (see [19]). For every endofunctor

$$
\text { final coalgebra }=\text { initial completely iterative algebra. }
$$


Remark 3.48. (a) Let $H$ be a finitary set functor, i.e., every element $x \in H A$ lies, for some finite subset $m: A^{\prime} \longrightarrow A$, in the image of $H m$. Then an algebra $a: H A \longrightarrow A$ is called iterative provided that for every equation morphism $e: X \longrightarrow H X+A$ with $X$ finite, there exists a unique solution $e^{\dagger}: X \longrightarrow A$.

This concept was studied for classical $\Sigma$-algebras by Nelson [21] and Tiurin [30], and for $\mathrm{H}$-algebras in general in [6].

(b) Form the colimit $C$, in Set, of the diagram of all finite coalgebras $a: A \longrightarrow H A$ with the colimit cocone $a^{+}: A \longrightarrow C$. Then there exists a unique morphism $c: C \longrightarrow H C$ with $c \cdot a^{+}=H^{+} \cdot a$. It was proved in [6] that $c$ is invertible and the resulting algebra is the initial iterative algebra for $H$.

Example 3.49 (see [6]). (a) The initial iterative algebra of $H X=X^{I} \times\{0,1\}$ consists of all finite minimal automata. This is isomorphic to its description as all regular languages.

(b) The initial iterative algebra of the finite power-set functor consists of all finite well-pointed graphs. See Section 4 for a description using rational trees.

Definition 3.50 (see [20]). A coalgebra is called locally finite if every element lies in a finite subcoalgebra.

Theorem 3.51 (see [20]). Let $H$ be a finitary set functor. Then initial iterative algebra $=$ final locally finite coalgebra.

Moreover, the final locally finite coalgebra is the colimit of all finite coalgebras in Coalg $H$.

Remark 3.52. We prove below that given a finitary set functor, the set of all finite wellpointed coalgebras forms the initial iterative algebra. For this result we do not need to assume (unlike the rest of this section) that the functor preserves intersections. This can be deduced from the following

Lemma 3.53. For every finitary set functor $H$ the Trnková closure $\bar{H}$ (see Remark 2.44) preserves (wide) intersections.

Proof. The functor $\bar{H}$ of Proposition 2.43 is obviously also finitary. It preserves finite intersections, and we deduce that it preserves all intersections. Given subobjects $m_{i}: A_{i}$ $\longrightarrow B(i \in I)$ with an intersection $m: A \longrightarrow B$, let $x \in \bar{H} B$ lie in the image of each $H m_{i}$; we are to prove that $x$ lies in the image of $\bar{H} m$. Choose a subset $n: C \longrightarrow B$ of the smallest (finite) cardinality with $x$ lying in the image of $\bar{H} n$. Since $\bar{H}$ preserves the intersection of $n$ and $m_{i}$, the minimality of $C$ guarantees that $n \subseteq m_{i}$ (for every $i \in I$ ). Thus, $n \subseteq m$, proving that $x$ lies in the image of $\bar{H} m$.

Notation 3.54. For every finitary set functor denote by

$$
\varrho H
$$

the set of all finite well-pointed coalgebras up to isomorphism.

Given a finite coalgebra $a: A \longrightarrow H A$ we again define a function

$$
a^{+}: A \longrightarrow \varrho H
$$

by assigning to every element $x: 1 \longrightarrow A$ the well-pointed coalgebra of Notation 3.18 ;

$$
a^{+}(x)=\mathrm{wp}(A, a, x) .
$$

This is well-defined due to Corollary 2.28 and Lemma 2.29 since $H$ and $\bar{H}$ have the same pointed coalgebras. 
Theorem 3.55. Every finitary set functor $H$ has the initial iterative algebra $\varrho H$.

Remark. $\varrho H$ has the canonical coalgebra structure

$$
\psi^{*}: \varrho H \longrightarrow H(\varrho H) .
$$

It assigns, analogously to (3.1), to every element $(A, a, x)$ of $\varrho H$ the following element of $H(\varrho H)$ :

$$
1 \stackrel{x}{\longrightarrow} A \stackrel{a}{\longrightarrow} H A \stackrel{\mathrm{Ha}^{+}}{\longrightarrow} H(\varrho H) .
$$

We prove below that this is the final locally finite coalgebra. Thus, $\varrho H$ is the initial iterative algebra w.r.t. the inverse of $\psi^{*}$, by Theorem 3.51 .

Proof. Analogously to the proof of Theorem 3.24 one verifies that the morphisms

$$
a^{+}:(A, a) \longrightarrow\left(\varrho H, \psi^{*}\right) \quad(A \text { finite })
$$

are coalgebra homomorphisms forming a cocone. By Remark 3.48(b) it remains to prove that this is a colimit in Coalg $H$. We verify that all $a^{+}$'s form a colimit cocone in Set. That is:

(i) Every element of $\varrho H$ has the form $a^{+}(x)$ for some finite coalgebra $(A, a)$ and some $x \in A$. Indeed, for every element $(A, a, x)$ of $\varrho H$ we have $a^{+}(x)=(A, a, x)$.

(ii) Whenever

$$
a^{+}(x)=b^{+}(y)
$$

holds for two finite coalgebras $(A, a)$ and $(B, b)$ and for elements $x \in A, y \in B$ (turning them into pointed coalgebras), there exists a zig-zag of homomorphisms of finite pointed coalgebras connecting $(A, a, x)$ with $(B, b, y)$. For that recall $a^{+}(x)=$ $\left(\bar{A}_{0}, \bar{a}_{0}, x_{0}\right)$ in the notation 3.18 , Here is the desired zig-zag:

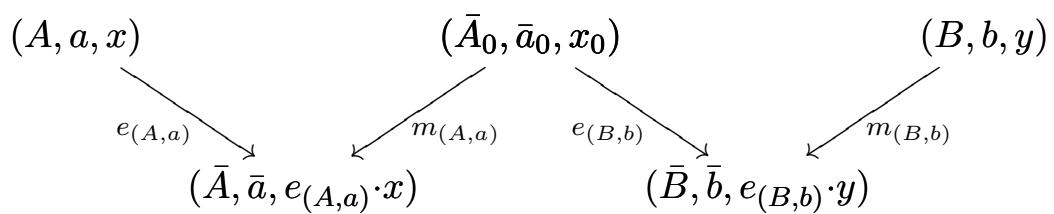

Remark 3.56. For non-finitary set functors $H$ the set $\varrho H$ also carries the structure above of a coalgebra. But this is in general not a fixed point of $H$. For example, the functor $H X=X^{\mathbb{N}}+1$ has the final coalgebra consisting of all countably branching trees. And $\varrho H$ is the set of all rational trees, i.e., those having only finitely many subtrees (up to isomorphism), see Example 4.28. This is a subcoalgebra of the final coalgebra, but not a fixed point of $H$.

Remark 3.57. Theorem 3.55] generalizes immediately to varieties of algebras that are locally finite, i.e. free algebras on finitely many variables are finite (for example, boolean algebras or semilattices).

Let $H$ be a finitary endofunctor preserving intersections. The set

$$
\rho H=\text { all finite well-pointed coalgebras }
$$

forms a subcoalgebra of the coalgebra of Construction 3.32. This is the initial iterative algebra for $H$. (The proof is entirely analogous to that of Proposition 3.34, based on Theorem 3.51 and the fact that a finite coproduct of finite coalgebras is finite.) 


\section{EXAMPles OF WELL-POINTEd COALGEBRAS}

For a number of important set functors $H$ we are going to apply the results of Section 3 and compare them to the well-known description of the three fixed points of interest: the final coalgebra, the initial algebra, and the initial iterative algebra (= final locally finite coalgebra). The last one is also called the rational fixed point of $H$. Throughout this section pointed coalgebras are considered up to (point-preserving) isomorphism. Recall that

$$
\begin{aligned}
& \nu H=\text { all well-pointed coalgebras } \\
& \mu H=\text { all well-founded, well-pointed coalgebras }
\end{aligned}
$$

and if $H$ is a finitary functor

$$
\varrho H=\text { all finite well-pointed coalgebras. }
$$

We are using various types of labeled trees throughout this section. Trees, too, are considered up to (label-preserving) isomorphism. Unless explicitly stated, trees are ordered, i.e., a linear ordering on the children of every node is always given.

In all our examples the endofunctors $H$ used preserves intersections and weak pullbacks. Recall from Rutten [23] that this implies that

(a) congruences on a coalgebra $A$ are precisely the kernel equivalences of homomorphisms $f: A \longrightarrow B$, and

(b) for every coalgebra the largest congruence is precisely the bisimilarity equivalence.

Also recall from Remark 3.20 that, for these functors, every pointed coalgebra yields a well-pointed one by first forming the "reachable part" and then the simple coreflection.

In pictures of pointed coalgebras the choice of the point $q_{0}$ is depicted by

$$
\rightarrow q_{0}
$$

\subsection{Moore automata.}

Given a set $I$ of inputs and a set $J$ of outputs, a Moore automaton on a set $Q$ (of states) is given by a next-state function $\delta: Q \times I \longrightarrow Q$ curried as

$$
\operatorname{curry} \delta: Q \longrightarrow Q^{I}
$$

an output function

$$
\text { out: } Q \longrightarrow J
$$

and an initial state $q_{0} \in Q$. The first two items form a coalgebra for

$$
H X=X^{I} \times J
$$

thus we work with pointed coalgebras for this functor, with $q_{0}$ as the chosen point. The behavior of an automaton is the function

$$
\beta: I^{*} \longrightarrow J
$$

which to every input word $w \in I^{*}$ assigns the output of the state reached from $q_{0}$ by applying the inputs in $w$. A function $\beta: I^{*} \longrightarrow J$ is called regular if the set of all functions $\beta(w-): I^{*} \longrightarrow J$ for $w \in I^{*}$ is finite. 
Lemma 4.1. The largest congruence on a Moore automaton merges states $q$ and $q^{\prime}$ iff by applying an arbitrary finite sequence of inputs to each of them, we obtain states with the same output.

This is well-known and easy to prove. Automata satisfying this condition are called simple. Another well-known fact is the following

Theorem 4.2. For every function $\beta: I^{*} \longrightarrow J$ there exists a reachable and simple Moore automaton with the behavior $\beta$. This automaton is unique up to isomorphism. It is finite iff $\beta$ is regular.

Corollary 4.3. For Moore automata, $H X=X^{I} \times J$, we have

$$
\begin{array}{ll}
\nu H \cong J^{I^{*}}, & \text { all functions } \beta: I^{*} \longrightarrow J ; \\
\varrho H \cong & \text { all regular functions } \beta: I^{*} \longrightarrow J ; \\
\mu H=\emptyset . &
\end{array}
$$

The coalgebra structure of $\nu H$ (and $\varrho H$ ) assigns to every $\beta: I^{*} \longrightarrow J$ the pair in $(\nu H)^{I} \times J$ consisting of the function $i \longmapsto \beta(i-)$ for $i \in I$ and the element $\beta(\varepsilon)$ of $J$.

Indeed, the isomorphism between $\nu H$, the set of all reachable and simple automata, and $J^{I^{*}}$ is given by the theorem above. And the structure map of Example 3.26 is easily seen to correspond to the map above, taking $\beta$ to $(i \longmapsto \beta(i-), \beta(\varepsilon))$. Also the isomorphism of $\varrho H$ and all regular functions follows from the theorem above; from Theorem 3.55 we know that $\varrho H$ is a subcoalgebra of $\nu H$.

Finally, $\mu H=\emptyset$ since no well-pointed coalgebra $(A, a)$ is well-founded due to the cartesian subcoalgebra

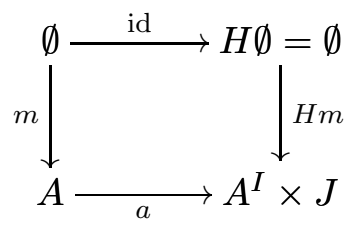

Example 4.4. If $J=\{0,1\}$ we get $\nu H=\mathscr{P} I^{*}$ and $\varrho H=$ regular languages, see Examples 3.28 and 3.49 (a).

\subsection{Mealy automata.}

For Mealy automata the next-state function has the form $\delta: Q \times I \longrightarrow Q \times J$ and in curried form this is a coalgebra for

$$
H X=(X \times J)^{I}
$$

Given a state $q$ of a Mealy automaton $Q$, its response function $f_{q}$ is the function $f_{q}: I^{\omega}$ $\longrightarrow J^{\omega}$ assigning to an infinite word of input symbols the infinite word of output symbols (delayed by one time unit) consisting of the outputs given by the transitions (as the computations of the inputs are performed, starting in $q$ ). Observe that $f_{q}$ is a causal function, i. e., for every infinite word $w$ the $n$-th component of $f_{q}(w)$ depends only on the first $n$ components of $w$.

Remark 4.5. Given a causal function $f: I^{\omega} \longrightarrow J^{\omega}$ the property above with $n=0$ tells us that the component 0 of $f(w)$ only depends on $w_{0}$. We thus obtain a derived function

$$
f^{0}: I \longrightarrow J
$$


with $f(i w)=f^{0}(i) w^{\prime}$ (for convenient $w^{\prime}$ ) for all $w \in I^{\omega}$.

Lemma 4.6. For every Mealy automaton the largest congruence merges precisely the pairs of states with the same response function.

Proof. Let $Q$ be a Mealy automaton, then the equivalence $q \sim q^{\prime}$ iff $f_{q}=f_{q^{\prime}}$ is obviously a congruence. We have a structure of a Mealy automaton $\bar{\delta}$ on $Q / \sim$ derived from that of $Q$ : Given a state $[q] \in Q / \sim$ and an input $i \in I$, the pair $\delta(q, i)=\left(q^{\prime}, j\right)$ yields $\bar{\delta}([q], i)=\left(\left[q^{\prime}\right], j\right)$. It is easy to verify that the canonical map $c: Q \longrightarrow Q / \sim$ is a coalgebra homomorphism $c:\left(Q, \delta, q_{0}\right) \longrightarrow\left(Q / \sim, \bar{\delta},\left[q_{0}\right]\right)$. Conversely, every congruence is contained in $\sim$ because given a coalgebra homomorphism $h: Q \longrightarrow \bar{Q}$ then for every state $q \in Q$ we have $f_{q}=f_{h(q)}$. Thus, the kernel congruence of $h$ is contained in $\sim$.

Corollary 4.7. The well-pointed Mealy automata are precisely those with an initial state $q_{0}$ such that the automaton is

(a) reachable: every state can be reached from $q_{0}$, and

(b) simple: different states have different response functions.

The automata satisfying (a) and (b) together are called "minimal". The following theorem can be found in Eilenberg [13, Theorem XII.4.1]:

Theorem 4.8. For every causal function $f$ there exists a unique well-pointed coalgebra whose initial state has the response function $f$.

Remark 4.9. Eilenberg also proves that a minimal Mealy automaton is finite iff $f$ has the property that the set of all functions $f(w-)$ where $w \in I^{*}$ is finite. Let us call such causal functions regular.

Corollary 4.10. For Mealy automata, $H X=(X \times J)^{I}$, we have

$$
\begin{aligned}
& \nu H \cong \text { all causal functions from } I^{\omega} \text { to } J^{\omega} \\
& \varrho H \cong \text { all regular causal functions } \\
& \mu H=\emptyset .
\end{aligned}
$$

The coalgebra structure of $\nu H$ (and that of $\varrho H$ ) assigns to every causal function $f: I^{\omega}$ $\longrightarrow J^{\omega}$ the map

$$
I \longrightarrow \nu H \times J, \quad i \longmapsto\left(f(i-), f^{0}(i)\right)
$$

for $f^{0}: I \longrightarrow J$ in Remark 4.5 .

Indeed, the first two statements follow from the theorem above, and the last one follows again from $H \emptyset=\emptyset$. The description of the final coalgebra is due to Rutten [24]. Eilenberg works with functions $f: I^{*} \longrightarrow J^{*}$ preserving length and prefixes, but it is immediate that these are just another way of coding all causal functions between infinite streams.

Remark 4.11. An alternative description of the final coalgebra for $H X=(X \times J)^{I}$ is:

$$
\nu H \cong J^{I^{+}}, \quad \text { all functions } \beta: I^{+} \longrightarrow J .
$$

Here and below, $I^{+}$is the set of finite non-empty words on the set $I$. The coalgebra structure assigns to every $\beta$ the mapping from $I$ to $\nu H \times J$ given by

$$
i \longmapsto(\beta(i-), \beta(i)) \quad \text { for } i \in I \text {. }
$$


Indeed, this coalgebra is isomorphic to that of all causal functions $f: I^{\omega} \longrightarrow J^{\omega}$ : to every function $\beta: I^{+} \longrightarrow J$ assign the causal function

$$
f\left(i_{0} i_{1} i_{2} \ldots\right)=\left(\beta\left(i_{0}\right), \beta\left(i_{0} i_{1}\right), \beta\left(i_{0} i_{1} i_{2}\right), \ldots\right) .
$$

\subsection{Streams.}

Consider the coalgebras for

$$
H X=X \times I+1 .
$$

Rutten [23] interprets them as dynamical systems with outputs in $I$ and with terminating states (where no next state is given). Every state $q$ yields a stream, finite or infinite, over $I$ by starting in $q$ and traversing the dynamical system as long as possible. We call it the response of $q$. It is an element of $I^{\omega}+I^{*}$.

Lemma 4.12. For a dynamical system the largest congruence merges two states iff they yield the same response.

Proof. Let $\sim$ be the equivalence from the statement of the lemma. Then we have an obvious dynamic system on $Q / \sim$, thus, $\sim$ is a congruence. Every coalgebra homomorphism $h: Q$ $\longrightarrow \bar{Q}$ fulfils: the response of $q$ and $h(q)$ is always the same. Therefore, $\sim$ is the largest congruence.

Corollary 4.13. A well-pointed coalgebra is a dynamical system with an initial state $q_{0}$ such that the system is

(a) reachable: every state can be reached from $q_{0}$, and

(b) simple: different states yield different responses.

\section{Example 4.14.}

(a) For every word $s_{1} \ldots s_{n}$ in $I^{*}$ we have a well-founded dynamic system

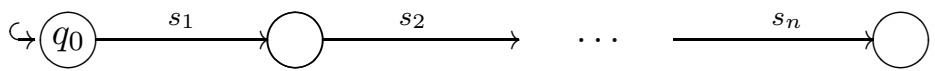

(b) For every eventually periodic stream in $I^{\omega}$,

$$
w=u v^{\omega} \quad \text { for } u, v \in I^{*},
$$

we have a pointed dynamical system

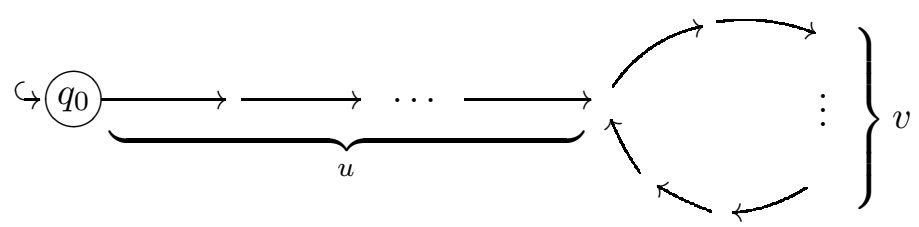

If we choose, for the given stream $w$, the words $u$ and $v$ of minimum lengths, then this system is well-pointed.

The following was already proved by Arbib and Manes [18, Theorem 10.2.5].

Corollary 4.15. For $H X=X \times I+1$ we have

$$
\begin{array}{ll}
\nu H \cong I^{*}+I^{\omega}, & \text { all finite and infinite streams }, \\
\varrho H \cong & \text { all finite and eventually periodic streams }, \\
\mu H \cong I^{*}, & \text { all finite streams. }
\end{array}
$$


The coalgebra structure assigns to every nonempty stream $w$ the pair

(tail $w$, head $w) \quad$ in $\nu H \times I$

and to the empty stream the right-hand summand of $H(\nu H)=\nu H \times I+1$.

Indeed, the description of $\nu H$ follows from Corollary 4.13 since by forming the response of $q_{0}$ we get a bijection between well-pointed coalgebras and streams in $I^{*}+I^{\omega}$. For the description of $\varrho H$ observe that a well-pointed system yields a finite or eventually periodic response iff it has finitely many states. The point in our statement is that $\mu H$ follows from the observation that a dynamical system is well-founded iff every run of a state is finite. Indeed, given a coalgebra $a: A \longrightarrow A \times I+1$, form the subset $m: A^{\prime} \longrightarrow A$ of all states with finite runs. We obtain a cartesian subcoalgebra

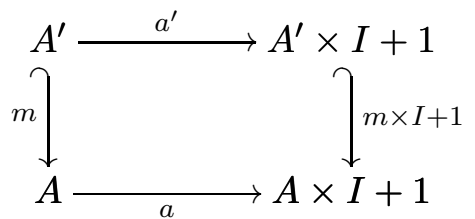

Thus, well-founded, well-pointed coalgebras are precisely those of Example 4.14(a).

\subsection{Binary trees.}

Coalgebras for the functor

$$
H X=X \times X+1
$$

are given, as observed by Rutten [23], by a set $Q$ of states which are either terminating or have precisely two next states according to a binary input, say $\{l, r\}$. Every state $q \in Q$ yields an ordered binary tree $T_{q}$ (i.e, nodes that are not leaves have a left-hand child and a right-hand one) by tree expansion: the root is $q$ and a node is either a leaf, if it is a terminating state, or has the two next states as children (left-hand for input $l$, right-hand for input $r$ ). Binary trees are considered up to isomorphism.

Lemma 4.16. For every system the largest congruence merges precisely the pairs of states having the same tree expansion.

Proof. Let $\sim$ be the equivalence with $q \sim q^{\prime}$ iff $T_{q}=T_{q^{\prime}}$. There is an obvious structure of a coalgebra on $Q / \sim$ showing that $\sim$ is a congruence. For every coalgebra homomorphism $h: Q \longrightarrow \bar{Q}$ the tree expansion of $q \in Q$ is always the same as the tree expansion of $h(q)$ in $\bar{Q}$. Thus $\sim$ is the largest congruence.

Corollary 4.17. A well-pointed system is a system with an initial state $q_{0}$ which is

(a) reachable: every state can be reached from $q_{0}$, and

(b) simple: different states have different tree expansions.

Moreover, tree expansion is a bijection between well-pointed coalgebras and binary trees (see Proposition 4.25 below). For instance, the dynamical system

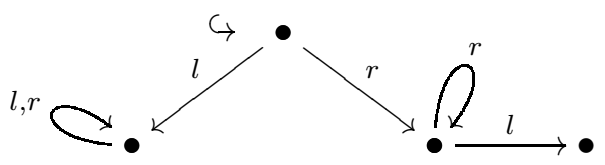


defines the tree

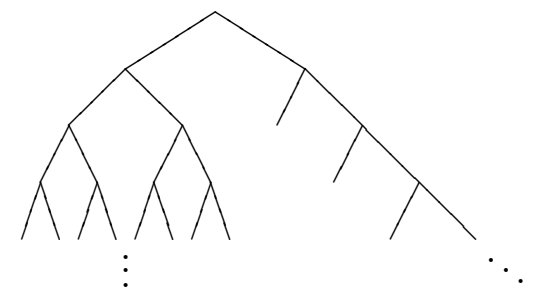

Observe that this tree has only 4 subtrees (up to isomorphism): this follows from the fact that the dynamical systems has 4 states. In general, the finite dynamical systems correspond to the rational trees, i.e., trees having (up to isomorphism) only finitely many subtrees. This description is due to Ginali [14].

Corollary 4.18. For the functor $H X=X \times X+1$ we have

$$
\begin{aligned}
& \nu H \cong \text { all binary trees } \\
& \varrho H \cong \text { all rational binary trees }, \\
& \mu H \cong \text { all finite binary trees. }
\end{aligned}
$$

The coalgebra structure is, in each case, the inverse of tree tupling: it assigns to the rootonly tree the right-hand summand of $\nu H \times \nu H+1$ and to any other tree the pair of its maximum subtrees.

Indeed, we only need to explain the last item $\mu H$. Given a coalgebra $a: A \longrightarrow A \times A+1$, let $m: A^{\prime} \longleftrightarrow A$ be the set of all states defining a finite subtree. This is a cartesian subcoalgebra

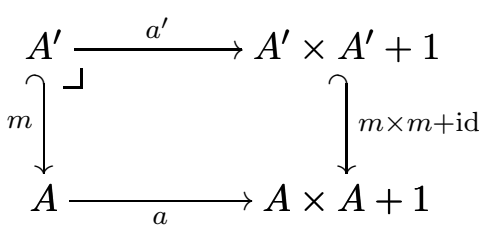

i.e., this square is a pullback: whenever a state $q \in A$ has both next states in $A^{\prime}$ or whenever $q$ is final, then $q \in A^{\prime}$. Thus, if $A$ is well-founded, then $A=A^{\prime}$. The converse implication is easy: recall the subsets $A_{i}^{*}$ of Notation 2.22, Here $A_{i}^{*}$ is the set of all states whose binary tree has depth at most $i$. Thus, if $A=A_{i}$ for some $i$, the initial state defines a tree of depth at most $i$.

\section{5. $\Sigma$-Algebras and $\Sigma$-coalgebras.}

All the examples above (and a number of other interesting cases) are subsumed in the following general case. Let $\Sigma$ be a signature, i.e., a set of operation symbols with given arities $\operatorname{ar}(s)$ of symbols $s \in \Sigma$; the arity is a natural number. The classical $\Sigma$-algebras are the algebras for the corresponding polynomial functor

$$
H_{\Sigma} X=\coprod_{\sigma \in \Sigma} X^{\operatorname{ar}(\sigma)}
$$

Coalgebras for $H_{\Sigma}$ are called $\Sigma$-coalgebras. 
Example 4.19. Let $I$ be a set of cardinality $n$. Deterministic automata $H X=X^{I} \times$ $\{0,1\}=X^{I}+X^{I}$ are given by two $n$-ary operations. Streams, $H X=X \times I+1$, are given by $n$ unary operations and a constant. Binary trees $H X=X \times X+1$ are given by one binary operation and one constant.

Definition 4.20. A $\Sigma$-tree is an ordered tree with nodes labeled in $\Sigma$ so that every node with $n$ children has a label of arity $n$. We consider $\Sigma$-trees up to isomorphism.

Observe that every $\Sigma$-tree $T$ is a coalgebra: the function $a: T \longrightarrow H_{\Sigma} T$ takes every node $x$ labelled by a symbol $\sigma \in \Sigma$ (of arity $n$ ) to the $n$-tuple $\left(x_{i}\right)_{i<n}$ of its children, an element of the $\sigma$-summand $T^{n}$ of $H_{\Sigma} T$.

In general a $\Sigma$-coalgebra $a: Q \longrightarrow H_{\Sigma} Q$ can be viewed as a system with a state set $Q$ labeled in $\Sigma$ :

$$
\bar{a}: Q \longrightarrow \Sigma
$$

and such that every state $q \in Q$ with $n$-ary label has "next states" forming an $n$-tuple

$$
a^{*}(q) \in Q^{n} \text {. }
$$

Indeed, to give a function $a: Q \longrightarrow H_{\Sigma} Q$ means precisely to given a pair $\left(\bar{a}, a^{*}\right)$ of functions as above.

Definition 4.21. Let $a: Q \longrightarrow H_{\Sigma} Q$ be a $\Sigma$-coalgebra.

(a) A computation of length $n$ is a word $i_{0} \cdots i_{n-1}$ in $\mathbb{N}^{*}$ for which there are states $q_{0}, \cdots, q_{n}$ in $Q$ with

$$
q_{k+1}=\text { the } i_{k} \text {-component of } a^{*}\left(q_{k}\right) \quad(k=0, \ldots, n-1) .
$$

(b) The tree expansion of a state $q$ is the $\Sigma$-tree

$$
T_{q}
$$

of all computations with initial state $q$. The label of a computation is $\bar{a}\left(q_{n}\right)$, where $q_{n}$ is its last state. And the children are all one-step extensions of that computation, i.e., all words $i_{0} \ldots i_{n-1} j$ with $j<\operatorname{ar}\left(\bar{a}\left(q_{n}\right)\right)$.

Lemma 4.22. The greatest congruence on a $\Sigma$-coalgebra merges precisely the pairs of states with the same tree expansion.

Proof. Let $\left(Q, \bar{a}, a^{*}\right)$ be a $\Sigma$-coalgebra and put $q \sim q^{\prime}$ iff $T_{q}=T_{q^{\prime}}$. Then we have a coalgebra structure on $Q / \sim$ : the label of $[q]$ is $\bar{a}(q)$, independent of the representative. The next-state $n$-tuple is $\left(\left[q_{i}\right]\right)_{i<n}$ where $a^{*}(q)=\left(q_{i}\right)$. It is easy to see that this is independent of the choice of representatives. And the quotient map is a coalgebra homomorphism from $Q$ to $Q / \sim$. Thus, $\sim$ is a congruence.

To prove that $\Sigma$ is the largest congruence, observe that given a coalgebra homomorphism $h: Q \longrightarrow Q^{\prime}$, then for every state $q \in Q$ we have $T_{q}=T_{h(q)}$. Indeed, an isomorphism $i: T_{q}$ $\longrightarrow T_{h(q)}$ is easy to define by induction on the depth of nodes of $T_{q}$. 
Corollary 4.23. Well-pointed $\Sigma$-coalgebras are the $\Sigma$-coalgebras with an initial state $q_{0}$ which are

(a) reachable: every state can be reached from $q_{0}$ by a computation, and

(b) simple: different states have different tree expansions.

Example 4.24. For every $\Sigma$-tree $T$ the equivalence on the nodes of $T$ given by

$$
x \sim y \quad \text { iff } \quad T_{x} \cong T_{y}
$$

where $T_{x}$ is the subtree of $T$ rooted at node $x$, is a congruence. And $T / \sim$ carries an obvious structure of a $\Sigma$-coalgebra. Let $[r]$ be the congruence class of the root, then the pointed $\Sigma$-coalgebra $(T / \sim,[r])$ is well-pointed.

Indeed, this pointed coalgebra is reachable: given a node $q$ of $T$ let $i_{0} \cdots i_{n-1}$ be the unique path from $r$ to $q$, then $i_{0} \cdots i_{n-1}$ is a computation in $T / \sim$ with initial state $[r]$ and terminal state $\left[q_{n}\right]$.

The simplicity of $T / \sim$ follows from Lemma 4.22 and the observation that the tree expansion of a state $[q]$ of $T / \sim$ is the subtree $T_{q}$ of $T$.

These are all well-pointed $\Sigma$-coalgebras:

Proposition 4.25. Every well-pointed coalgebra is isomorphic to $(T / \sim,[r])$ for a unique $\Sigma$-tree T.

Proof. It is well-known that the coalgebra $\tau: B \longrightarrow H_{\Sigma} B$ of all $\Sigma$-labeled trees where $\tau$ is given by

$$
\begin{aligned}
\bar{\tau}(T) & =\text { label of the root of } T, \text { and } \\
\tau^{*}(T) & =\left(T_{i}\right)_{i<n},
\end{aligned}
$$

where $T_{i}$ is the $i$-th maximum subtree is final. Indeed, for every coalgebra $(Q, a)$ the unique coalgebra homomorphism $h: Q \longrightarrow B$ is given by tree expansion (see Definition 4.21): $h(q)=T_{q}$.

Now from Theorem 3.24 we have the final coalgebra $\nu H_{\Sigma}$ of all well-pointed coalgebras. The tree expansion map $h: \nu H_{\Sigma} \longrightarrow B$ is then an isomorphism. The inverse $h^{-1}$ takes a tree $T$ to the coalgebra $(T / \sim,[r])$ above: this is immediate from the fact that the tree expansion of $[r]$ in $T / \sim$ is $T$.

Proposition 4.26. A $\Sigma$-coalgebra is well-founded iff all its tree-expansions are well-founded $\Sigma$-trees, i.e., $\Sigma$-trees with no infinite path.

Proof. Given a $\Sigma$-coalgebra $A$ let $m: A^{\prime} \longleftrightarrow A$ be the subset of all states $q \in A$ with $T_{q}$ well-founded. This is, obviously, a subcoalgebra. And it is cartesian

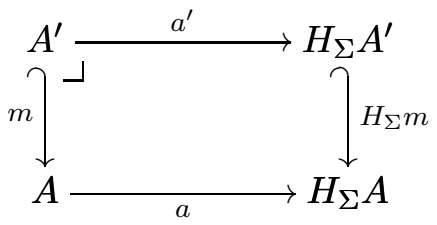

Indeed, if a state $q$ has the property that all components of $a^{*}$ lies in $A^{\prime}$, the $q$ lies in $A^{\prime}$. Thus $A$ is well-founded iff $A=A^{\prime}$. 
Definition 4.27 (see [14]). A $\Sigma$-tree is called rational if it has up to isomorphism only finitely many subtrees.

Example 4.28. Given a finite $\Sigma$-coalgebra, all tree expansions of its states are rational.

Indeed, if $Q=\left\{q_{1}, \ldots, q_{n}\right\}$ is the state set, then every subtree of $T_{q_{i}}$ (given by a computation with initial state $q_{i}$ ) has the form $T_{q_{j}}$ : take $q_{j}$ to be the terminal state of the computation.

Corollary 4.29. For every finitary signature $\Sigma$ we have

$$
\begin{aligned}
& \nu H_{\Sigma} \cong \text { all } \Sigma \text {-trees } \\
& \varrho H_{\Sigma} \cong \text { all rational } \Sigma \text {-trees } \\
& \mu H_{\Sigma} \cong \text { all finite } \Sigma \text {-trees. }
\end{aligned}
$$

The coalgebra structure is in each case inverse to tree-tupling.

Indeed, the isomorphism between $\nu H_{\Sigma}$ and all $\Sigma$-trees is given by Proposition 4.25. And the coalgebra structure of Remark 3.25 corresponds to the inverse of tree-tupling, i.e., it assigns to a $\Sigma$-tree $T$ with $a^{*}(r)=\left(x_{1}, \ldots, x_{n}\right)$ the $n$-tuple $\left(T_{x_{1}}, \ldots, T_{x_{n}}\right)$ in the $\sigma$-summand of $H_{\Sigma}\left(\nu H_{\Sigma}\right)$ where $\sigma$ is the label of the root.

Finally, the isomorphism between $\varrho H_{\Sigma}$ (all finite well-pointed coalgebras) and rational $\Sigma$-trees follows from Proposition 4.25 and Example 4.28, The last item follows from König's Lemma: every well-founded finitely branching tree is finite.

Example 4.30. For the functor $H X=X^{*}$ we can use nonlabeled trees: we have

$$
\begin{aligned}
& \nu H \cong \text { all finitely branching trees } \\
& \varrho H \cong \text { all rational finitely branching trees } \\
& \mu H \cong \text { all finite trees. }
\end{aligned}
$$

Indeed, let $\Sigma$ be the signature with one $n$-ary operation for every $n \in \mathbb{N}$. Then $H_{\Sigma} X \cong$ $X^{*}$. And $\Sigma$-trees need no labeling, since operations already differ by arities.

\subsection{Graphs.}

Here we investigate coalgebras for the power-set functor $\mathscr{P}$. In the rest of Section 4 all trees are understood to be non-ordered. That is, a tree is a directed graph with a node (root) from which every node can be reached by a unique path.

Recall the concept of a bisimulation between graphs $X$ and $Y$ : it is a relation $R \subseteq X \times Y$ such that whenever $x R y$ then every child of $x$ is related to a child of $y$, and vice versa. Two nodes of a graph $X$ are called bisimilar if they are related by a bisimulation $R \subseteq X \times X$.

Lemma 4.31. The greatest congruence on a graph merges precisely the bisimilar pairs of states.

This follows, since $\mathscr{P}$ preserves weak pullbacks, from general results of Rutten [23].

Corollary 4.32. A pointed graph $\left(G, q_{0}\right)$ is well-pointed iff it is

(a) reachable: every vertex can be reached from $q_{0}$ by a directed path, and

(b) simple: all distinct pairs of states are non-bisimilar. 
Example 4.33. Aczel [1] introduced the canonical picture of a (well-founded) set $X$. It is the graph with vertices all sets $Y$ such that a sequence

$$
Y=Y_{0} \in Y_{1} \in \cdots \in Y_{n}=X
$$

of sets exists. The neighbors of a vertex $Y$ are all of its elements. When pointed by $X$, this is a well-pointed graph. Indeed, reachability is clear. And suppose $R$ is a bisimulation and $Y R Y^{\prime}$, then we prove $Y=Y^{\prime}$. Assuming the contrary, there exists $Z_{0} \in Y$ with $Z_{0} \notin Y^{\prime}$, or vice versa. Since $R$ is a bisimulation, from $Z_{0} \in Y$ we deduce that $Z_{0}^{\prime} \in Y^{\prime}$ exists with $Z_{0} R Z_{0}^{\prime}$. Clearly $Z_{0} \neq Z_{0}^{\prime}$. Thus, we substitute $\left(Y, Y^{\prime}\right)$ by $\left(Z_{0}, Z_{0}^{\prime}\right)$ and obtain $Z_{1} \in Z_{0}$ and $Z_{1}^{\prime} \in Z_{0}^{\prime}$ with $Z_{1} R Z_{1}^{\prime}$ but $Z_{1} \neq Z_{1}^{\prime}$ etc. This is a contradiction to the well-foundedness of $X$ : we get an infinite sequence $Z_{n}$ with

$$
\cdots Z_{2} \in Z_{1} \in Z_{0} \in Y \text {. }
$$

Here are some concrete examples of canonical pictures and their corresponding tree expansions (cf. Remark 4.34 below):

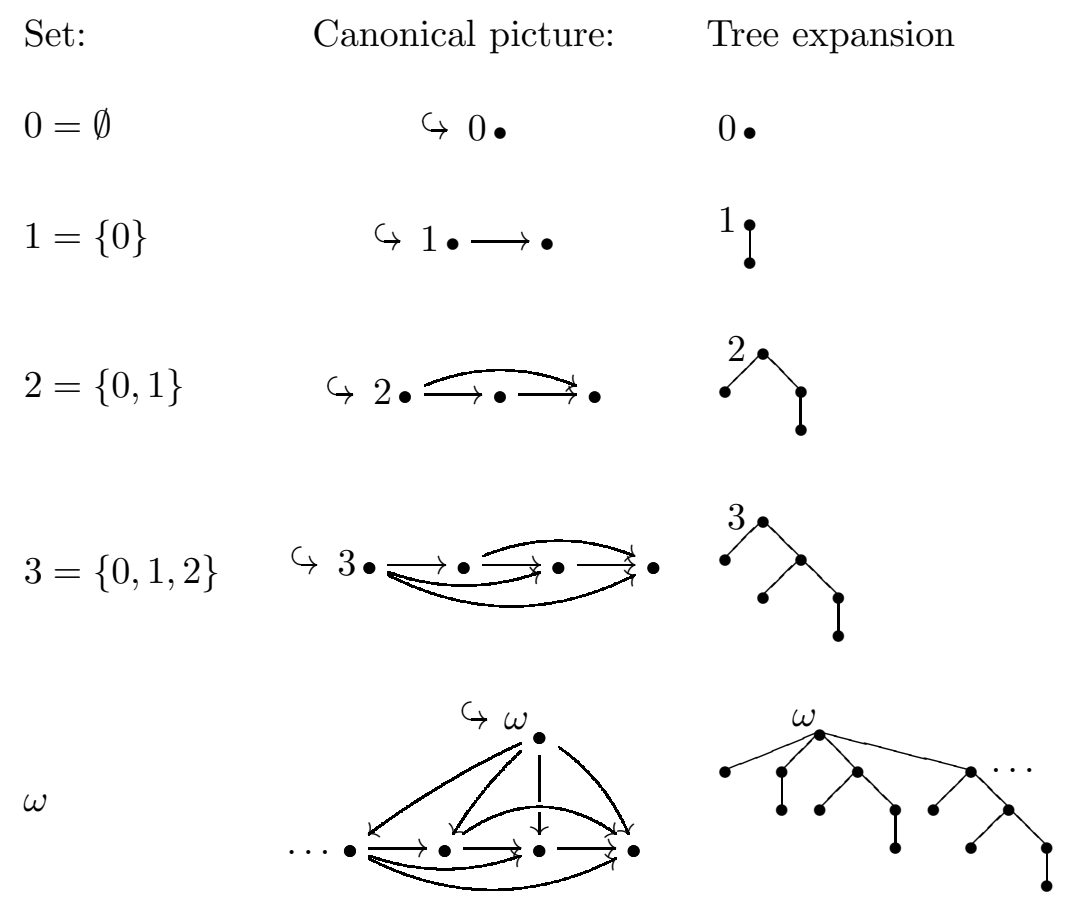

Remark 4.34. Given a vertex $q$ of a graph, its tree expansion is (similarly to the ordered case, see Definition 4.21) the non-ordered tree

$T_{q}$

whose nodes are all finite directed paths from $q$.

The children of a node $p$ are all one-step extensions of the path $p$. The root is $q$ (considered as the path of length 0 ).

For every pointed graph $(G, x)$ the tree expansion is the tree $T_{x}$. In the previous example we saw tree expansions of the given pointed graphs.

Definition 4.35 (Worrell [33]). By a tree-bisimulation between trees $T_{1}$ and $T_{2}$ is meant a graph bisimulation $R \subseteq T_{1} \times T_{2}$ which 
(a) relates the roots,

(b) $x_{1} R x_{2}$ implies that $x_{1}$ and $x_{2}$ are the roots or have related parents, and

(c) $x_{1} R x_{2}$ implies that the depths of $x_{1}$ and $x_{2}$ are equal.

A tree $T$ is called strongly extensional iff every tree bisimulation $R \subseteq T \times T$ is trivial: $R \subseteq \Delta_{T}$.

Example 4.36. The tree expansion of a well-pointed graph $\left(G, q_{0}\right)$ is strongly extensional. Indeed, given a tree bisimulation $R \subseteq T_{q_{0}} \times T_{q_{0}}$, we obtain a graph bisimulation $\bar{R} \subseteq G \times G$ consisting of all pairs $\left(q_{1}, q_{2}\right)$ of vertices for which paths $p_{i}$ from $q_{0}$ to $q_{i}$ exist, $i=1,2$, with $p_{1} R p_{2}$. Since $G$ is simple, $\bar{R} \subseteq \Delta$. Thus, for all pairs $\left(p_{1}, p_{2}\right)$ of paths:

if $p_{1} R p_{2}$ then the last vertices of $p_{2}$ and $p_{1}$ are equal.

We prove $p_{1} R p_{2}$ implies $p_{1}=p_{2}$ by induction on the maximum $k$ of the lengths of $p_{1}$ and $p_{2}$. For $k=0$ we have $p_{1}=q_{0}=p_{2}$. For $k+1$ we have $p_{1}^{\prime} R p_{2}^{\prime}$ where $p_{i}^{\prime}$ is the trimming of $p_{i}$ by one edge (since $R$ is a tree bisimulation). Then $p_{1}^{\prime}=p_{2}^{\prime}$ implies $p_{1}=p_{2}$ because the last vertices are equal.

Furthermore, there are no other extensional trees:

Proposition 4.37. Every strongly extensional tree is the tree expansion of a unique (up to isomorphism) well-pointed graph.

Proof. Let $T$ be a strongly extensional tree with root $r$, considered as a coalgebra for $\mathscr{P}$.

(a) Existence. The coalgebra $(T / \sim,[r])$ where $\sim$ merges bisimilar vertices of $T$ is wellpointed by Lemma 4.31. Its tree expansion $T^{\prime}=(T / \sim)_{[r]}$ is (isomorphic to) the given tree $T$. Indeed, the relation $R \subseteq T \times T^{\prime}$ of all pairs $(x, p)$ where $x$ is a node of $T$ and $p$ is the equivalence class of the unique path from $r$ to $x$ is clearly a tree bisimulation. Since $\mathscr{P}$ preserves weak pullbacks, it follows that the composite $R \circ R^{-1}$ of $R$ and $R^{-1}$ is also a tree bisimulation, see [23]. But $T$ is strongly extensional, thus $R \circ R^{-1} \subseteq \Delta$. Also $T^{\prime}$ is strongly extensional, see Example 4.36, thus $R^{-1} \circ R \subseteq \Delta$. Since for every $x$ there is a pair $(x, p)$ in $R$, we conclude that $R$ is (the graph of) an isomorphism from $T$ to $T^{\prime}$.

(b) Uniqueness: If well-pointed graphs $\left(G, q_{0}\right)$ and $\left(G^{\prime}, q_{0}^{\prime}\right)$ have isomorphic tree expansions, then they are isomorphic. Arguing analogously to (a) we only need to find a graph bisimulation $R \subseteq G \times G^{\prime}$ and use the simplicity of $G$ and $G^{\prime}$. For that, we just observe that there is a graph bisimulation between $\left(G, q_{0}\right)$ and $T_{q_{0}}$ : the relation $R \subseteq G \times T_{q_{0}}$ of all pairs $(q, p)$ where $q \in G$ is the last vertex of the path $p$ from $q_{0}$ to $q$.

Corollary 4.38. $\nu \mathscr{P}=$ all strongly extensional trees.

We must be careful here: $\mathscr{P}$ has no fixed points. But recall the extension of set functors to classes in Remark 3.29, For $\mathscr{P}$ this is the functor $\mathscr{P}^{*} X=\{A ; A$ is a set with $A \subseteq X\}$. Its (large) final coalgebra is the coalgebra of all (small) strongly extensional trees.

Notation 4.39. Let $\mathscr{P}_{\lambda}$ be the subfunctor of all subsets of cardinality less than $\lambda$. (Thus $\mathscr{P}_{\omega}$ is the finite power-set functor.) Then by precisely the same argument as above one proves

Corollary 4.40. For every cardinal $\lambda$,

$$
\nu \mathscr{P}_{\lambda}=\text { all } \lambda \text {-branching strongly extensional trees. }
$$


This was proved for $\lambda=\omega$ by Worrell [33] and for general $\lambda$ by Schwencke [26]. Our proof is entirely different.

We know from Example 2.10(1) that the well-founded graphs are precisely the graphs without an infinite directed path. Now strong extensionality can, in the case of well-founded trees, be simplified to extensionality which says that for every node different children define non-isomorphic subtrees. Thus we get

Corollary 4.41. $\quad \mu \mathscr{P}=$ all well-founded, extensional trees; $\mu \mathscr{P}_{\lambda}=$ all $\lambda$-branching, well-founded, extensional trees.

Analogously to Example 4.28 the rational fixed point of the finite-powerset functor $\mathscr{P}_{\omega}$ consists of all rational strongly extensional trees, i.e., those with finitely many subtrees up to isomorphism:

Corollary 4.42. For the finite power-set functor $\mathscr{P}_{\omega}$ we have

$$
\begin{aligned}
\nu \mathscr{P}_{\omega} & =\text { all finitely branching, strongly extensional trees, } \\
\varrho \mathscr{P}_{\omega} & =\text { all finitely branching, rational, strongly extensional trees, and } \\
\mu \mathscr{P}_{\omega} & =\text { all finite extensional trees. }
\end{aligned}
$$

\subsection{Sets and non-well-founded sets.}

We revisit $\mu \mathscr{P}$ and $\nu \mathscr{P}$ here from a set-theoretic perspective. Before coming to the nonwell-founded sets, let us observe that Example 4.33 has the following strengthening:

Lemma 4.43. Well-founded, well-pointed graphs are precisely the canonical pictures of well-founded sets.

This follows from the standard fact from set theory that every well-pointed graph $G$ has a unique Mostowski collapse, also called a decoration in Aczel [1, see Introduction], i.e., coalgebra homomorphism $d$ to the class Set of sets considered as a graph with $\in$ as the neighborhood relation. That is, $d$ assigns to every vertex $x$ a set $d(x)$ as follows:

$$
d(x)=\{d(y) ; y \in G \text { a neighbor of } x\} .
$$

Observe that the kernel of $d$ is clearly a congruence on $G$. Thus, given a well-pointed, wellfounded graph $\left(G, q_{0}\right)$, we know that $d$ is monic. From that it follows that the canonical picture of the set $d\left(q_{0}\right)$ is isomorphic to $\left(G, q_{0}\right)$.

Corollary 4.44. $\mu \mathscr{P}=$ the class of all sets.

This was proved by Rutten and Turi in [25. The bijection between well-founded, wellpointed graphs and sets (given by the canonical picture) takes the finite graphs to the hereditarily finite sets, i.e., finite sets with finite elements which also have finite elements, etc. More precisely: a set $X$ is hereditarily finite if all sets in the canonical picture of $X$ are finite:

Corollary 4.45. $\mu \mathscr{P}_{\omega}=$ all hereditarily finite sets.

In order to describe the final coalgebra for $\mathscr{P}$ in a similar set-theoretic manner, we must move from the classical theory to the non-well-founded set theory of Aczel [1]. Nonwell-founded set theory is obtained by swapping the axiom of foundation, telling us that (Set, $\in$ ) is well-founded, with the following

Anti-foundation axiom. Every graph has a unique decoration. 
Example 4.46. The decoration of a single loop is a set $\Omega$ such that $\Omega=\{\Omega\}$.

The coalgebra (Set, $\in$ ) where now Set is the class of all non-well-founded sets, is of course final for $\mathscr{P}$ : the decoration of any graph $G$ is the unique homomorphism $d: G$ $\longrightarrow$ Set.

Corollary 4.47. In the non-well-founded set theory

$$
\nu \mathscr{P}=\text { all sets. }
$$

Let us turn to the finite power-set functor $\mathscr{P}_{\omega}$. Its final coalgebra consists of all sets whose canonical picture is finitely branching. They are called 1-hereditarily finite, notation $H F^{1}[\emptyset]$, in the monograph of Barwise and Moss [9]. The rational fixed point of $\mathscr{P}_{\omega}$ consists of all sets whose canonical picture is finite, they are called 1/2-hereditary in [9]. The collection of these sets is denoted by $H F^{1 / 2}[\emptyset]$. For well-founded sets (with canonical picture well-founded) the two collections coincide.

Corollary 4.48. In the non-well-founded set theory

$$
\begin{aligned}
& \nu \mathscr{P}_{\omega}=H F^{1}[\emptyset], \quad \text { the } 1 \text {-hereditarily finite sets, } \\
& \varrho \mathscr{P}_{\omega}=H F^{1 / 2}[\emptyset], \quad \text { the } 1 / 2 \text {-hereditarily finite sets, and } \\
& \mu \mathscr{P}_{\omega}=\text { the well-founded, hereditarily finite sets. }
\end{aligned}
$$

\subsection{Labeled transition systems.}

Here we consider, for a set $A$ of actions, labeled transition systems (LTS) as coalgebras for $\mathscr{P}(-\times A)$. A bisimulation between two labeled transition systems $G$ and $G^{\prime}$ is a relation $R \subseteq G \times G^{\prime}$ such that

$$
\text { if } \begin{aligned}
x R x^{\prime} & \text { then for every transition } x \stackrel{a}{\longrightarrow} x^{\prime} \text { in } G \text { there exists } \\
& y^{\prime} \in G^{\prime} \text { and a transition } y \stackrel{a}{\longrightarrow} y^{\prime} \text { with } x^{\prime} R y^{\prime} \text {, and } \\
& \text { vice versa. }
\end{aligned}
$$

States $x, y$ of an LTS are called bisimilar if $x R y$ for some bisimulation $R \subseteq G \times G$.

Lemma 4.49. For every LTS the greatest congruence merges precisely the bisimilar pairs of states.

This, again, follows from general results of Rutten [23] since $\mathscr{P}(-\times A)$ preserves weak pullbacks.

Corollary 4.50. An LTS together with an initial state $q_{0}$ is well-pointed iff it is

(a) reachable: every state can be reached from $q_{0}$ (by a sequence of actions), and

(b) simple: distinct states are non-bisimilar.

The tree expansion of a state $q$ is a (non-ordered) tree with edges labeled in $A$, shortly, an $A$-labeled tree. For $A$-labeled trees we modify Definition 4.35 and speak about tree bisimulation if a bisimulation $R \subseteq T_{1} \times T_{2}$ also fulfils (a)-(c) of Definition 4.35. An $A$ labeled tree $T$ is strongly extensional iff every tree bisimulation $R \subseteq T \times T$ is trivial.

Proposition 4.51. Tree expansion is a bijection between well-pointed LTS and strongly extensional A-labeled trees.

The proof is analogous to that of Proposition 4.37. Also the rest is analogous to the case of $\mathscr{P}$ above: 
Corollary 4.52. $\quad \nu \mathscr{P}(-\times A) \cong$ all strongly extensional A-labeled trees, $\nu \mathscr{P}_{\lambda}(-\times A) \cong$ all $\lambda$-branching, strongly extensional A-labeled trees.

Corollary 4.53. For the finitely branching LTS we have

$\nu \mathscr{P}_{\omega}(-\times A) \cong$ all finitely branching, strongly extensional A-labeled trees, $\varrho \mathscr{P}_{\omega}(-\times A) \cong$ all rational, finitely branching strongly extensional A-labeled trees, $\mu \mathscr{P}_{\omega}(-\times A) \cong$ all finite extensional A-labeled trees.

\section{Conclusion}

For functors $H$ satisfying the (mild) assumption of preservation of intersections we described (a) the final coalgebra as the set of all well-pointed coalgebras, (b) the initial algebra as the set of all well-pointed coalgebras that are well-founded, and (c) in the case where $H$ is finitary, the initial iterative algebra as the set of all finite well-pointed coalgebras. This is based on the observation that given an element of a final coalgebra, the subcoalgebra it generates has no proper subcoalgebras nor proper quotients - shortly, this subcoalgebra is well-pointed. And different elements define non-isomorphic well-pointed subcoalgebras. We then combined this with our result that for all set functors the initial algebra is precisely the final well-founded coalgebra. (For set functors preserving inverse images this was proved by Taylor [28].) More generally, for endofunctors of varieties preserving intersections we proved that the final coalgebra is carried by the sets of all well-pointed coalgebras, and the initial algebra is carried by the set of all well-founded, well-pointed coalgebras, and we presented a concrete description. Numerous examples demonstrate that this view of final coalgebras and initial algebras is useful in applications.

More generally, for functors preserving finite intersections the fact that initial algebras coincide with final well-founded coalgebras was proved in locally finitely presentable categories. The description of the final coalgebra was formulated concretely only in varieties

of algebras. In future research we intend to generalize this result to a wider class of base categories.

\section{REFERENCES}

[1] P. Aczel, Non-well-founded Sets, CSLL Lect. Notes. 14, Stanford CSLI Publications, Stanford 1988.

[2] P. Aczel and N. Mendler, A final coalgebra theorem, Lect. N. Comput. Sci. (1989), 357-365.

[3] J. Adámek, Free algebras and automata realizations in the language of categories, Comment. Math. Univ. Carolinae 15 (1974), 589-602.

[4] J. Adámek, H. Herrlich and G. E. Strecker, Abstract and Concrete Categories: The Joy of Cats, 2nd edition, Dover Publications, 2009.

[5] J. Adámek, D. Lücke and S. Milius, Recursive coalgebras of finitary functors, Theoret. Inform. Appl. 41 (2007), 442-462.

[6] J. Adámek, S. Milius and J. Velebil, Iterative algebras at work, Math. Struct. Comput. Sci. 16 (2006), 1085-1131.

[7] J. Adámek, S. Milius and J. Velebil, On coalgebra based on classes, Theoret. Comput. Sci. 316 (2004), $3-23$.

[8] J. Adámek and J. Rosický, Locally Presentable and Accessible Categories, Cambridge University Press, 1994.

[9] J. Barwise and L. S. Moss, Vicious Circles, CSLI Publications, Stanford 1996.

[10] M. M. Bonsangue, S. Milius and A. Silva, Sound and complete axiomatizations of coalgebraic language equivalence, available at http://arxiv.org/abs/1104.2803, 2011. 
[11] J. Brzozowski and H. Tamm, Theory of Átomata, Proc. 15th International Conference on Developments in Language Theory (DLT'11), ser. Lecture Notes Comput. Sci., vol. 6795. Springer, 2011, pp. 105-116.

[12] V. Capretta, T. Uustalu and V. Vene, Recursive coalgebras from comonads, Inform. and Comput. 204 (2006), 437-468.

[13] S. Eilenberg, Automata, Languages and Machines, Vol. A, Academic Press, New York 1974.

[14] S. Ginali, Regular trees and the free iterative theory, J. Comput. System Sci. 18 (1979), 228-242.

[15] P. Gabriel and F. Ulmer, Lokal präsentierbare Kategorien, Lect. N. Mathem. 221, Springer-Verlag Berlin, 1971.

[16] H.-P. Gumm, On minimal coalgebras, Appl. Cat. Str. 16 (2008), 313-332.

[17] H.-P. Gumm, From T-coalgebras to filter structures and transition systems, CALCO 2005, Lect. N. Comput. Sci. 3629 (2005), 194-212.

[18] E. G. Manes and M. A. Arbib, Algebraic approaches to program semantics, Springer-Verlag, New York, 1986.

[19] S. Milius, Completely iterative algebras and completely iterative monads, Inform. and Comput. 196 (2005), 1-41.

[20] S. Milius, A sound and complete calculus for finite stream circuits, Proc. 25th Annual Symposium on Logic in Computer Science (LICS'10), IEEE Computer Society 2010, 449-458

[21] E. Nelson, Iterative algebras, Theoret. Comput. Sci. 25 (1983), 67-94.

[22] G. Osius, Categorical set theory: a characterization of the category of sets, J. Pure Appl. Algebra 4 (1974), 79-119.

[23] J. J. M. M. Rutten, Universal coalgebra: a theory of systems, Theoret. Comput. Sci. 249 (2000), 3-80.

[24] J. J. M. M. Rutten, Algebraic specification and coalgebraic sybnthesis of Mealy automata, Elect. N. Theoret. Comput. Sci. 160 (2006), 305-319.

[25] J. J. M. M. Rutten and D. Turi, On the foundations of final semantics: non-standard sets, metric spaces, partial orders, Lect. N. Comput. Sci. 666, Springer 1993, 477-530.

[26] D. Schwencke, Coequational logic for accessible functors, Inform. Comput. 208 (2010), 1469-1489.

[27] A. Tarski, A lattice theoretical fixed point theorem and its applications, Pacific J. Math. 5 (1955), 285-309.

[28] P. Taylor, Towards a unified treatment of induction I: the general recursion theorem, preprint 1995-6, see www.paultaylor.eu/ordinals/\#towuti.

[29] P. Taylor, Practical Foundations of Mathematics, Cambridge University Press, 1999.

[30] J. Tiurin, Unique fixed points vs. least fixed points, Theoret. Comput. Sci. 12 (1980), 229-254.

[31] V. Trnková, On a descriptive classification of set functor I, Comment. Math. Univ. Carolinae 12 (1971), 323-352.

[32] V. Trnková, J. Adámek, V. Koubek and J. Reiterman, Free algebras, input processes and free monads, Comment. Math. Univ. Carolinae 16 (1975), 339-351.

[33] J. Worrell, On the final sequence of a finitary set functor, Theoret. Comput. Sci. 338 (2005), 184-199. 